Mixed-Use

Commercial \& Residential

Podium Building

$263077^{\text {th }}$ Ave. SE

Mercer Island 98040

Washington State

Gene Voloshenko

FPE 596

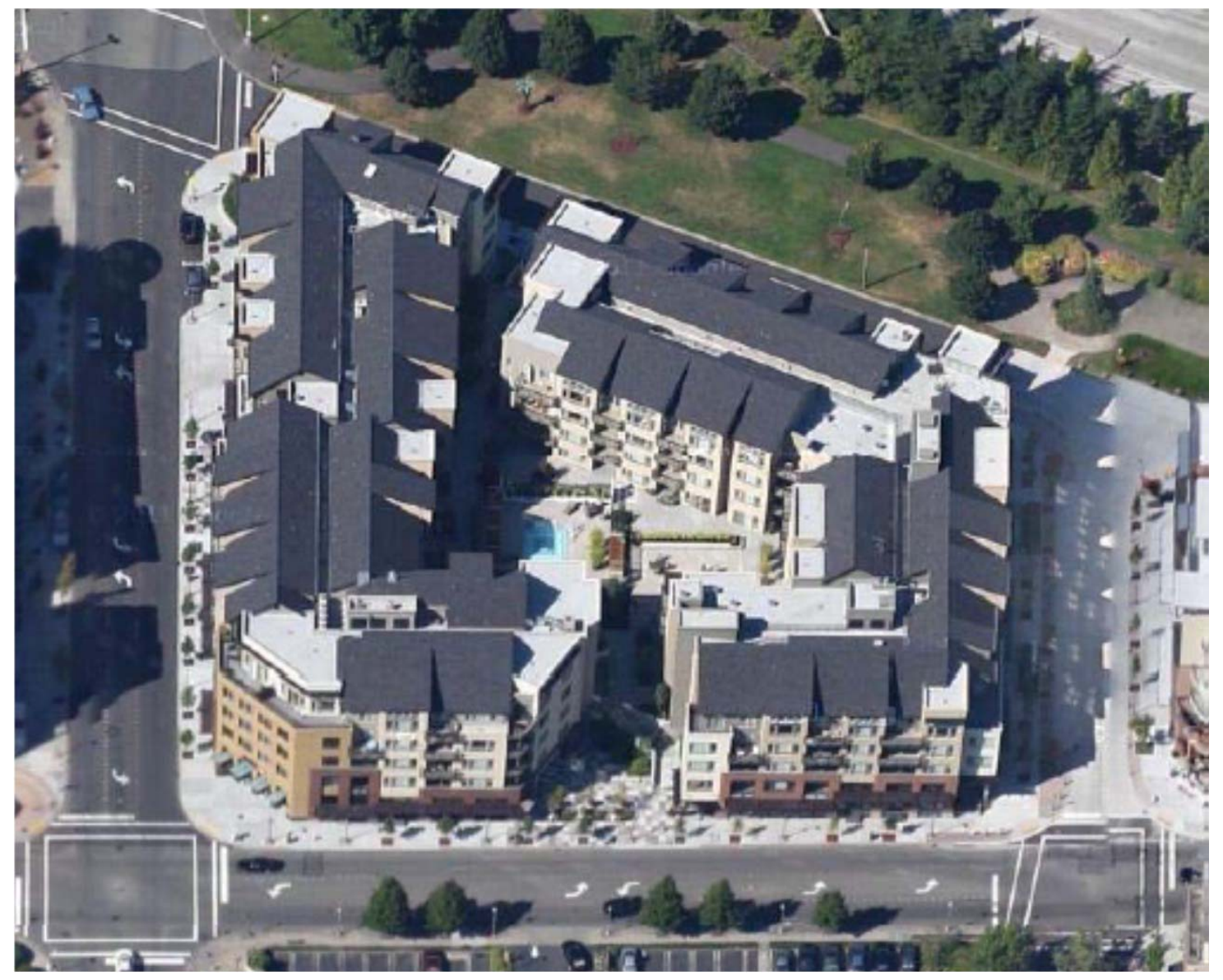




\section{Mixed-Use Commercial/Residential}

Podium Building

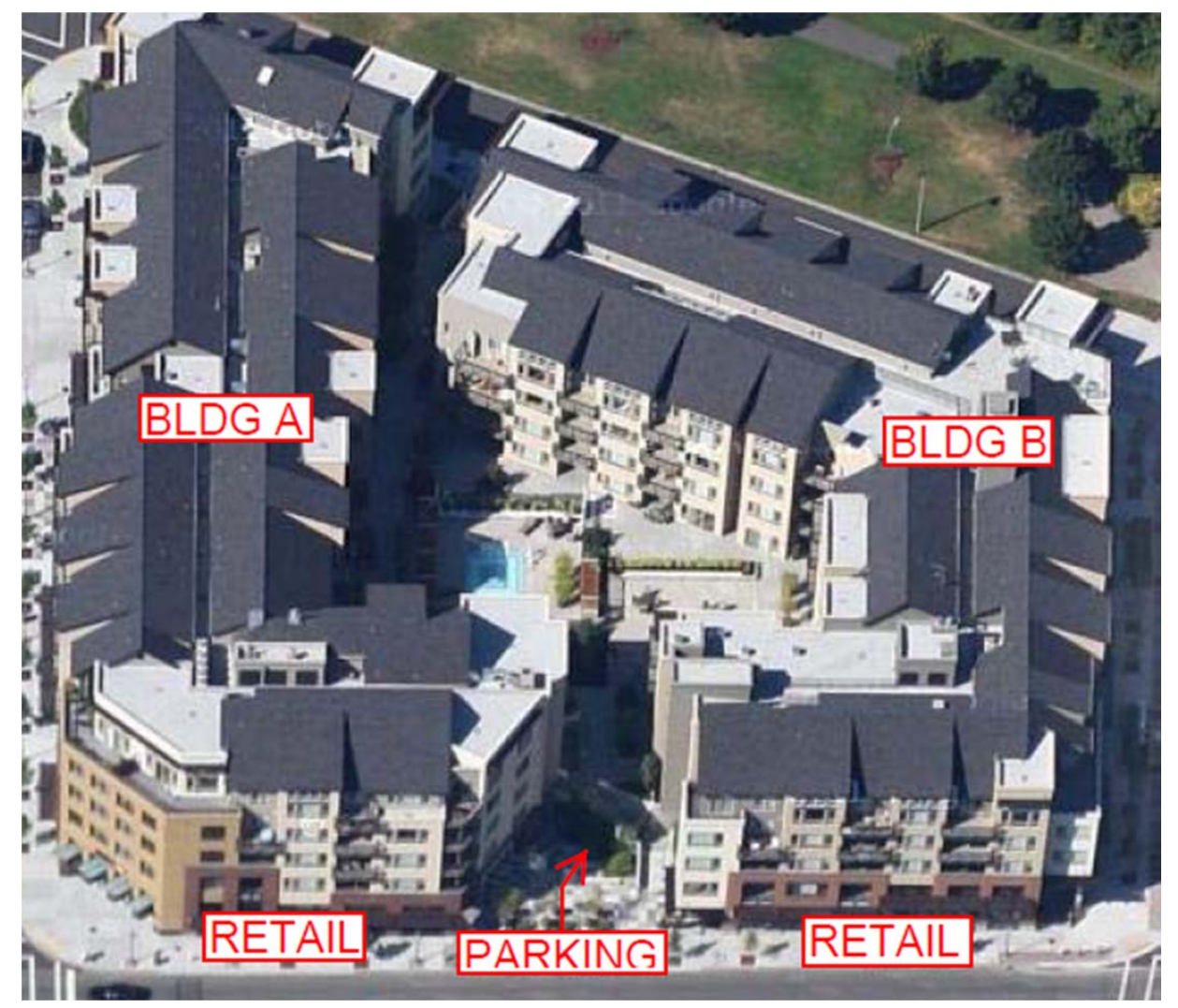

Two residential 4-storey wood-framed buildings on top of the non-combustible podium structure

Bldg $A=90$ units

Bldg $B=95$ units

Bldg: Parking/Retail

- Level 1/ P1 (same elevation)

- 6 Commercial Retail Units + Amenity

- Visitor parking

- $\quad$ Level P0 Residential parking

Constructed $\sim 2007 / 08$

Common multi-family construction in the Northwest United States

Involved in the design of a sprinkler system for this project

(Design/Built project) 
77 Central - Front view (Bldg A)

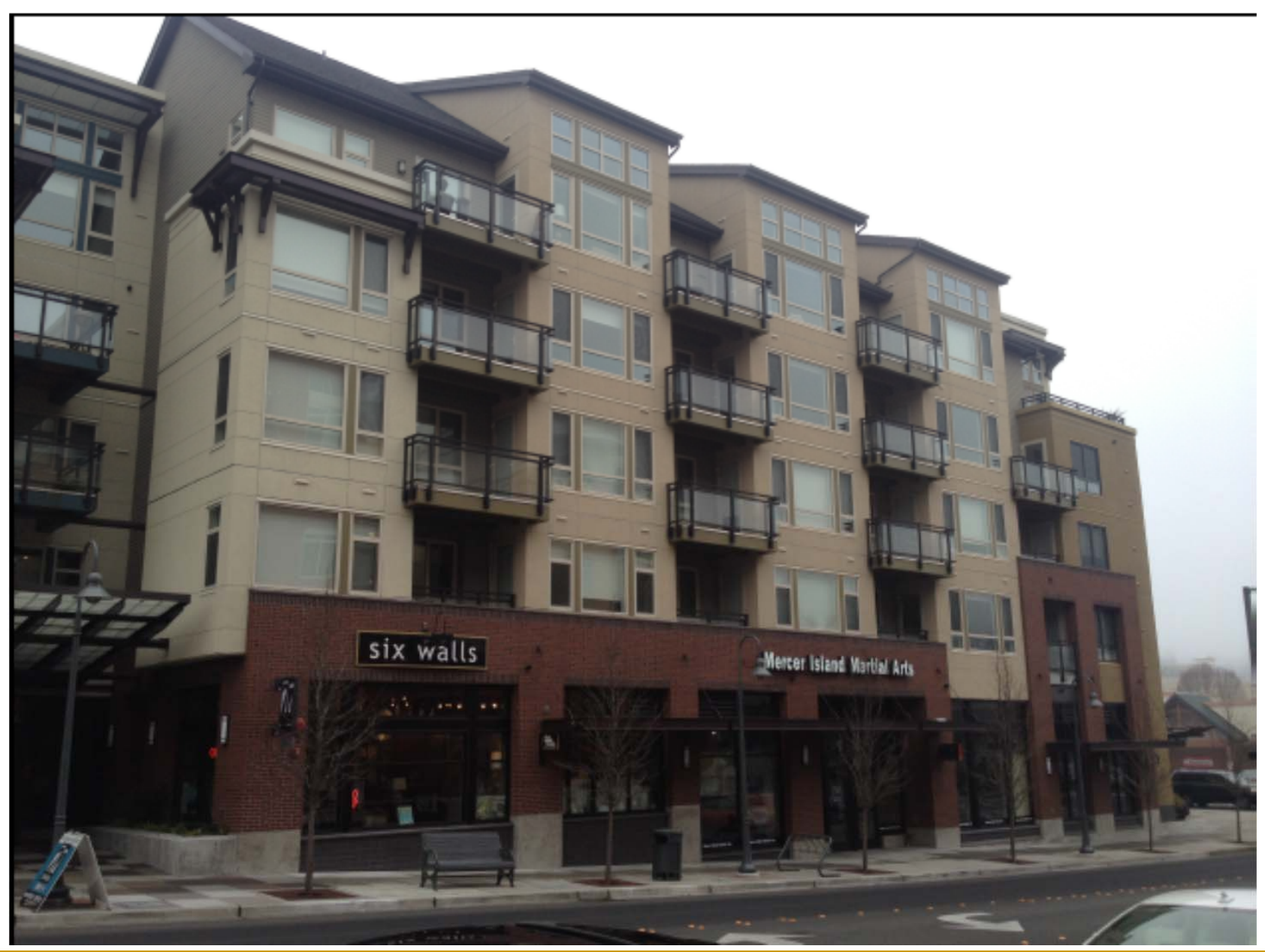


77 Central - Side view (Bldg B)

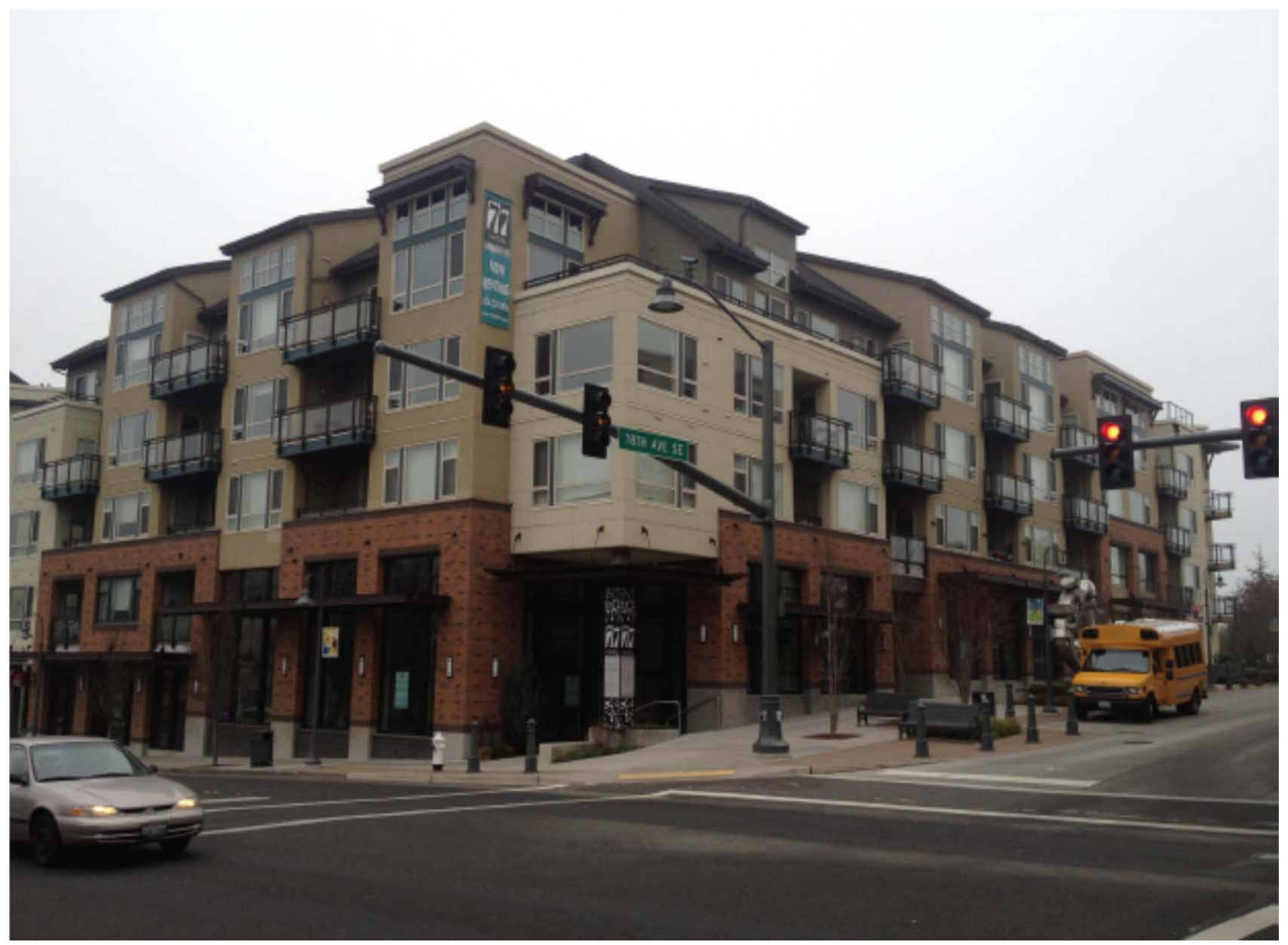




\section{PRESENTATION OUTLINE}

- Building construction requirements

- Discuss separation of buildings

- 3-hour horizontal fire barrier

- Code mark-up drawings review

- Fire Department Access plan (site plan)

- Structural fire protection components

- Travel distances to exits

- Egress Analysis

- Prescriptive (Code)

- Phased evacuation concept

- Fire Protection Systems:

- Fire Sprinkler System (NFPA 13)

- $\quad$ Fire Alarm System (NFPA 72)

- Performance - Based Approach

- Garage car fire in Parking P1 (Open door to Amenity space)

口 $\quad$ Fire in the Amenity Room

a Kitchen fire on the top level unit (Mezzanine configuration)

- Recommendations 


\section{Central Complex}

The building type is commonly referred to as a podium building, however, this term does not appear in the IBC.

This building type separates the buildings above and below a required 3-hour horizontal assembly into 3 separate and distinct buildings specifically for the purposes of:

- determining area limitations,

- continuity of fire walls,

- limitation of number of stories, and

- type of construction only for wood-framed buildings on top of podium.

(For all other building requirements this is still one building because two residential structures are interconnected by a common parking garage. Example: Sprinkler system, fire alarm system, exterior cladding, etc)

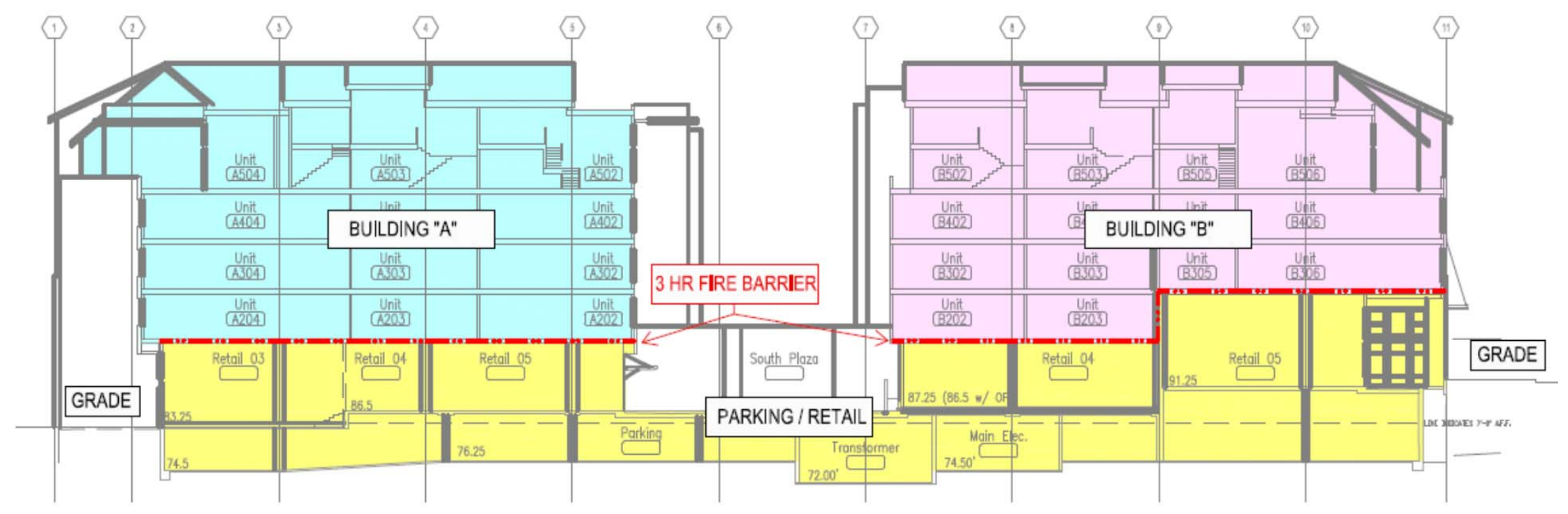




\section{Central Complex:}

- Building 1 - Residential Building A

- $\quad$ Building 2 - Residential Building B

- Building 3 - Parking and Retail Building

Bldg Separation: Complex is subdivided into three separate buildings in accordance with IBC 2000 Section 508.2 for "Group S-2 enclosed parking garage with Groups A, B, M or R above". This will allow for combustible construction of residential structures (Group R) above the Parking/Retail Building. 3-hr fire resistance rating is required.

Reference 508.2 typically refers to Group S-2 (parking garage) building under the 3-hr podium for separation of buildings purposes. However, a number of exceptions are permitted as below:

- Group A with less than 300 persons, and

- Group B or Group M are permitted provided that the entire structure below the horizontal assembly having a minimum 3-hour fire-resistance rating and is protected by an approved automatic sprinkler system. 


\section{Building Construction:}

\section{(Residential Bldg A \& B) -}

- Major Occupancies

- IBC Requirement

- Construction Type

- Floor Fire Ratings

- Roof Fire Rating

- Building Height

- Total Building Height

- Building Areas:

- Bldg A

口 Bldg B

- Sprinkler System

- Fire Alarm System

- Standpipe System
- $\quad$ Residential R2 - Apartment Building

- $\quad$ Type III A

- $\quad$ Combustible Construction Permitted (Section 508.2)

- $\quad 1 \mathrm{~h}$ - Residential to Residential

$3 \mathrm{~h}$ - Residential to Parking garage

$1 \mathrm{hr}$

4 Storeys permitted (from Podium. Ref. 508.2)

5 Storeys from grade level

$24,870 \mathrm{ft}^{2}\left(29,160 \mathrm{ft}^{2}\right.$ permitted-modified)

$24,709 \mathrm{ft}^{2}\left(29,536 \mathrm{ft}^{2}\right.$ permitted-modified)

Required (NFPA 13) - 5 Storey bldgs from grade level

Required (NFPA 72)

Required (NFPA 14)

Note: $\quad$ Building area (footprint) for Bldgs A and B exceed the requirements of the code. Therefore area modification for each building will be applied as follows:

- next slide 


\section{Building Construction - Modification}

GENERAL BUILDING HEIGHTS AND AREAS:

TABLE $503-504.1$

\begin{tabular}{|c|c|c|c|c|c|c|c|c|c|c|}
\hline & & \multicolumn{7}{|c|}{$\begin{array}{l}\text { TABLE 503-continued } \\
\text { ALLOWABLE HEIGHT AND BUILDING AREAS } \\
\text { Height limitations shown as stories and feet above grade plane. } \\
\text { Area limitations as determined by the definition of "Area, building", per floor. }\end{array}$} & & \\
\hline & & \multicolumn{9}{|c|}{ TYPE OF CONSTRUCTION } \\
\hline & & \multicolumn{2}{|c|}{ TYPE I } & \multicolumn{2}{|c|}{ TYPE I } & \multicolumn{2}{|c|}{ TYPE III } & \multirow{2}{*}{$\frac{\text { TYPE IV }}{\text { HT }}$} & \multicolumn{2}{|c|}{ TYPEV } \\
\hline & & A & B & A & B & A & B & & $\bar{A}$ & B \\
\hline & & UL & 160 & 65 & 55 & 65 & 55 & 65 & 50 & 40 \\
\hline$M$ & $\begin{array}{l}\mathrm{S} \\
\mathrm{A}\end{array}$ & $\begin{array}{l}\text { UL } \\
\text { UL }\end{array}$ & $\begin{array}{l}11 \\
\text { UL }\end{array}$ & 21.500 & 12.500 & $\begin{array}{c}4 \\
18,500\end{array}$ & 12,500 & $\stackrel{4}{40,500}$ & $\begin{array}{c}3 \\
14,000\end{array}$ & $\begin{array}{c}1 \\
9.000\end{array}$ \\
\hline R-1 & $\begin{array}{l}\mathrm{S} \\
\mathrm{A}\end{array}$ & $\begin{array}{l}\text { UL } \\
\text { UL }\end{array}$ & $\begin{array}{l}11 \\
\text { UL }\end{array}$ & 24.000 & $\stackrel{4}{4}, 000^{-}$ & $\begin{array}{c}4 \\
24000\end{array}$ & $\begin{array}{c}4 \\
16,000\end{array}$ & $\stackrel{4}{40,500}$ & $\stackrel{3}{3}, 000$ & $\begin{array}{c}2 \\
7,000\end{array}$ \\
\hline R-2 $2^{a}$ & $\begin{array}{l}S \\
A\end{array}$ & $\begin{array}{l}\text { UL. } \\
\text { UL }\end{array}$ & $\begin{array}{l}11 \\
\text { UL }\end{array}$ & 24,000 & $\begin{array}{c}4 \\
16,000\end{array}$ & $\begin{array}{c}4 \\
24,000\end{array}$ & $16 \stackrel{4}{4}$ & $\begin{array}{c}4 \\
20,500\end{array}$ & 12.000 & $\stackrel{2}{7.000}$ \\
\hline R.32 & $\begin{array}{l}\text { S } \\
\text { A }\end{array}$ & $\begin{array}{l}\text { UL } \\
\text { UL }\end{array}$ & $\begin{array}{l}11 \\
\text { UL }\end{array}$ & $\stackrel{4}{\text { UL }}$ & $\stackrel{4}{\text { UL }}$ & $\begin{array}{l}4 \\
\text { UL }\end{array}$ & $\begin{array}{c}4 \\
\text { UL }\end{array}$ & $\begin{array}{c}4 \\
\text { UL }\end{array}$ & $\stackrel{3}{\mathrm{UL}}$ & $\begin{array}{c}3 \\
\text { UL. }\end{array}$ \\
\hline & $s$ & $\mathrm{rn}$ & 11 & 1 & $A$ & $A$ & $A$ & $A$ & 3 & ? \\
\hline
\end{tabular}

- Modifications Permitted as Following:

- Section 506.1 permits for Building Area modification based on frontage increase and for provision of an Automatic Sprinkler system

- Bldg A (modified area) $=29,160$ sq.ft permitted

- $\quad$ Bldg $B$ (modified area) $=29,536$ sq.ft permitted

- Existing Bldg $A$ and $B$ are not more than 25,000 sq.ft each. 


\section{Alternative Construction Types (Discussion Only!)}

GENERAL BUILDING HEIGHTS AND AREAS:

TABLE $503-504.1$

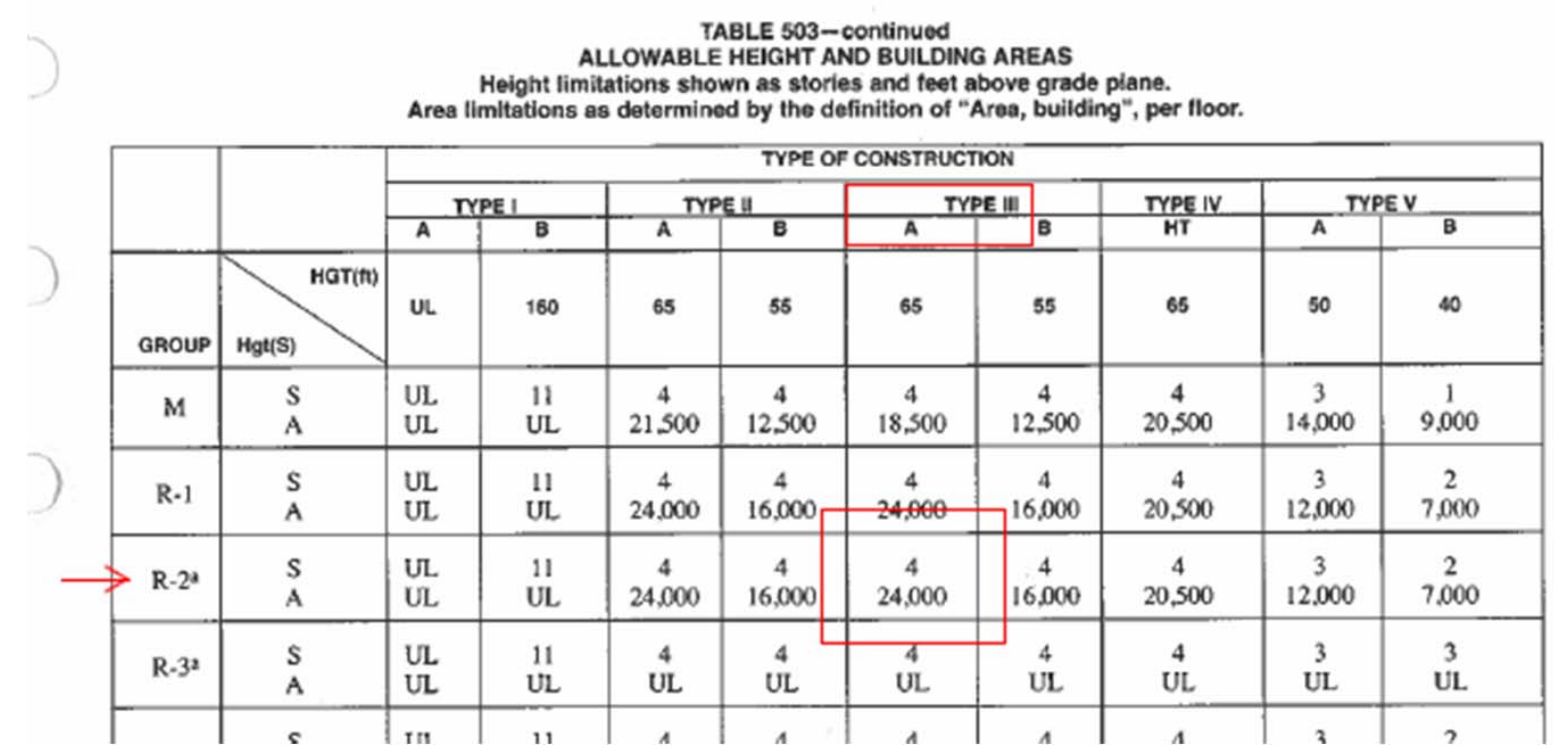

Construction Types:

- Type III (B) = limited by building area of 16,000 sq.ft

- Type IV = heavy timber (not applicable)

- $\quad$ Type $V(A)=$ limited by building area of 12,000 sq.ft 


\section{Building Construction:}

\section{(Parking/Retail) -}

- Major Occupancies

- IBC Requirement

- Construction Type

- Floor Fire Ratings

- Structural frame

- Podium Fire Rating

- Building Height

- Building Area

- Sprinkler System

- Fire Alarm System

- Standpipe System
Group S2 - Parking Garage

Group M - Mercantile

Group A2 - Assembly (Amenity)

Type I A (Ref. 508.2)

- Non-combustible

- $\quad 2 \mathrm{~h}$ between lower and upper parking levels

- $\quad 3$ h columns

- $\quad 3$ h - Parking Garage to Residential

- $\quad$ One underground and one above ground levels

- $\quad 66,300$ sq.ft (Unlimited permitted)

- $\quad$ Required (NFPA 13)

- $\quad$ Required (NFPA 72)

- $\quad$ Required (NFPA 14)

Note: $\quad 3 h$ Slab (Post-Tensioned)

FT (Fire and Temperature) rating required (IBC 2000 Sec. 711.4.1.2) 


\section{Code Mark-up Drawings}

- Fire Department Access Plan (site plan)

- Structural Fire Protection

- fire barriers and separations

- Travel distances to exits

- $\quad 2 \frac{1}{2}$ " hose connections and hose stations locations 


\section{Fire Department Access Plan - Site}

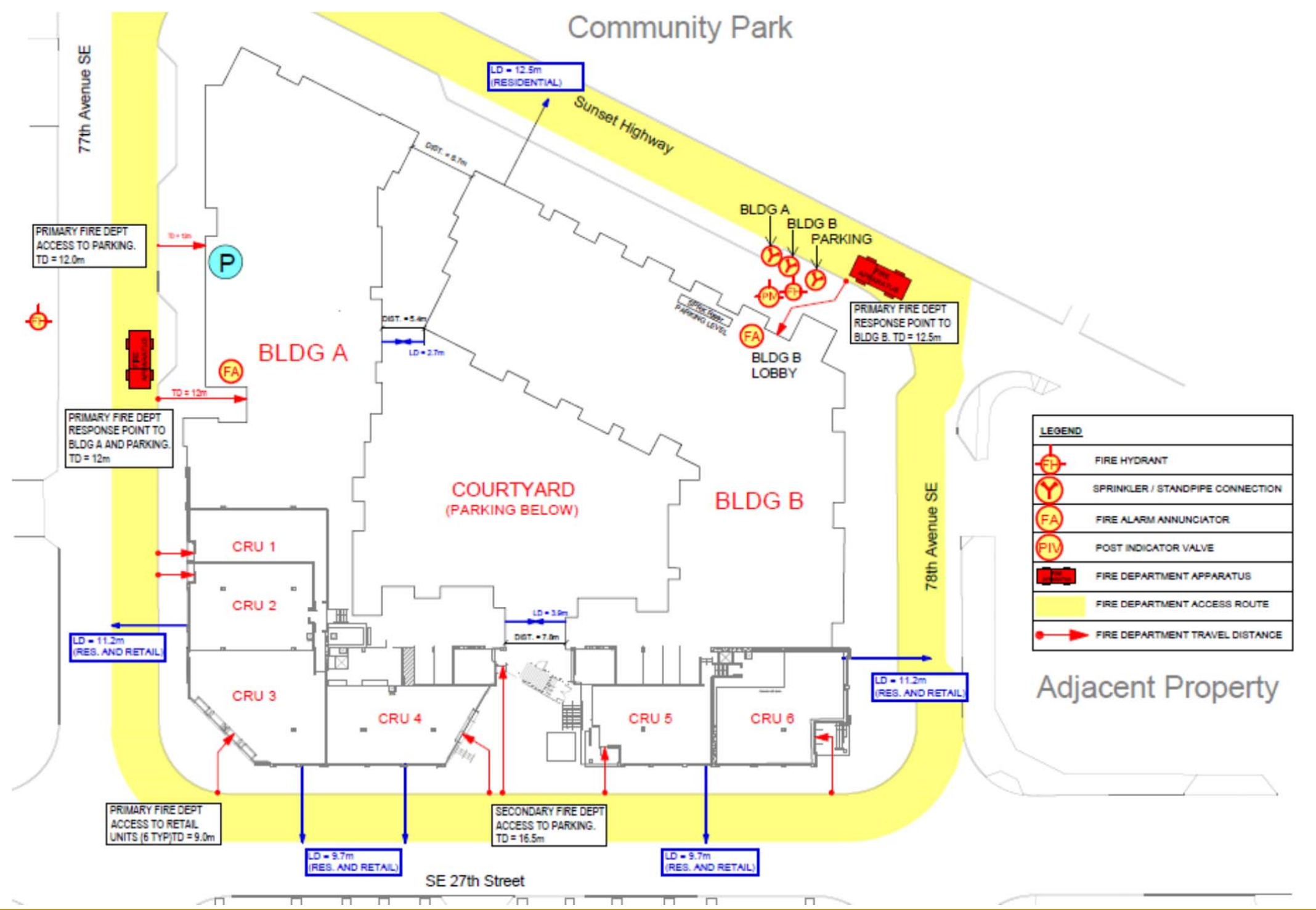




\section{Table 601 Structural Requirement (Type IA) Parking/Retail Bldg}

\begin{tabular}{|c|c|c|c|c|c|c|c|c|c|}
\hline \multirow[b]{2}{*}{ BULLDING ELEMENT } & \multicolumn{2}{|c|}{$>$ TYPEI } & \multicolumn{2}{|c|}{ TYPE ॥ } & \multicolumn{2}{|c|}{ TYPE III } & \multirow{2}{*}{$\begin{array}{c}\text { TYPE IV } \\
\text { HT }\end{array}$} & \multicolumn{2}{|c|}{ TYPE V } \\
\hline & A & B & $A^{d}$ & B & $A^{d}$ ) & B & & $A^{d}$ & B \\
\hline $\begin{array}{l}\text { Structural frame }{ }^{2} \\
\text { Including columns, girders, trusses }\end{array}$ & $3 b$ & $2^{b}$ & I & 0 & 1 & 0 & HT & 1 & 0 \\
\hline $\begin{array}{l}\text { Bearing walls } \\
\text { Exterior } \\
\text { Interior }\end{array}$ & $\begin{array}{c}3 \\
3 b\end{array}$ & $\frac{2}{2 b}$ & $\begin{array}{l}1 \\
1\end{array}$ & $\begin{array}{l}0 \\
0\end{array}$ & $\begin{array}{l}2 \\
1\end{array}$ & $\begin{array}{l}2 \\
0\end{array}$ & $\stackrel{2}{1 / \mathrm{HT}}$ & $\begin{array}{l}1 \\
1\end{array}$ & $\begin{array}{l}0 \\
0\end{array}$ \\
\hline $\begin{array}{l}\text { Nonbearing walls and partitions } \\
\text { Exterior } \\
\text { Interiore }\end{array}$ & & \multicolumn{8}{|c|}{$\begin{array}{c}\text { See Table } 602 \\
\text { See Section } 602\end{array}$} \\
\hline $\begin{array}{l}\text { Floor construction } \\
\text { Including supporting beams } \\
\text { and joists }\end{array}$ & 2 & 2 & I & 0 & 1 & 0 & HT & 1 & 0 \\
\hline $\begin{array}{l}\text { Roof construction } \\
\text { Including supporting beams } \\
\text { and joists }\end{array}$ & $11 / 2^{c}$ & Ic & $1^{c}$ & $\theta^{c}$ & 10 & 0 & HT & IE & 0 \\
\hline
\end{tabular}

Note:

Roof construction is indicated as $1 \frac{1 / 2}{2}-\mathrm{hr}$, for this project a 3-hr fire barrier will be constructed in accordance with Section 508.2 


\section{3-h Columns to Support a 3-h Fire Barrier}

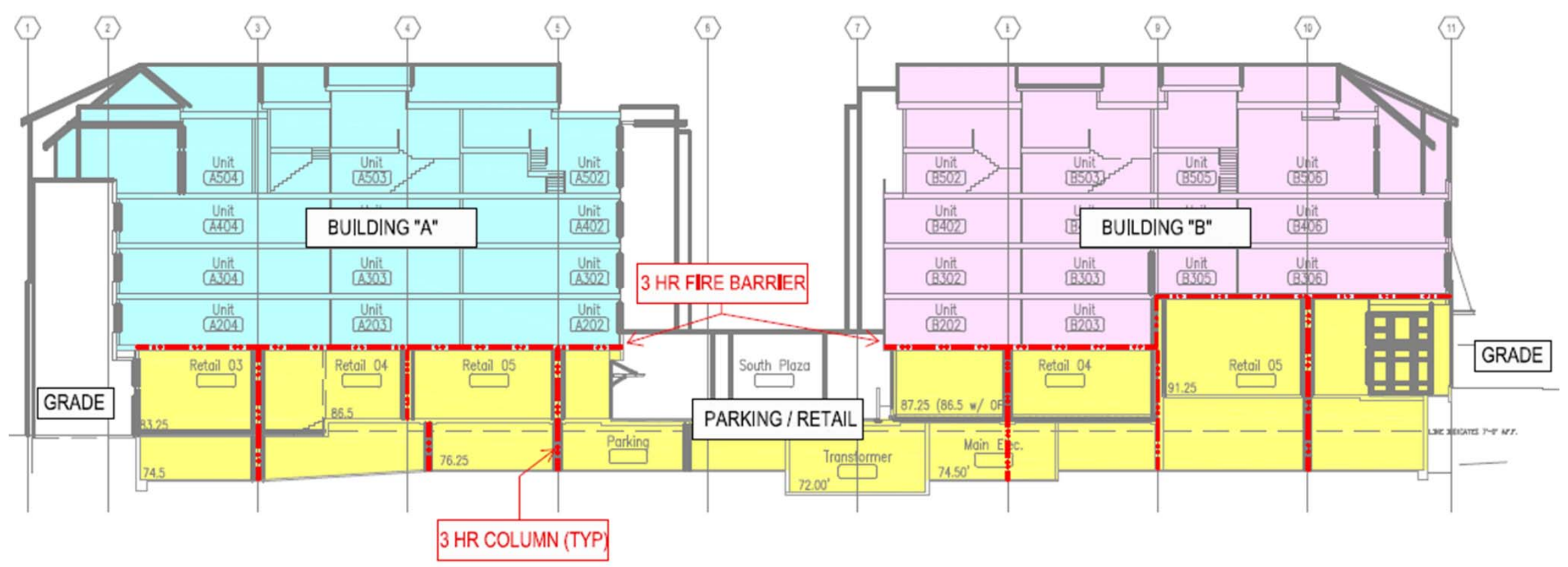




\section{Table 601 Structural Requirements (Type III A) Residential Bldg A and B}

TABLE 601

FIRE-AESISTANCE RATING REQUIREMENTS FOR BUILDING ELEMENTS (hours)

\begin{tabular}{|c|c|c|c|c|c|c|c|c|c|}
\hline \multirow[b]{2}{*}{ BULLDING ELEMENT } & \multicolumn{2}{|c|}{ TYPE I } & \multicolumn{2}{|c|}{ TYPE \| } & \multicolumn{2}{|c|}{$>$ TYPE III } & \multirow{2}{*}{$\begin{array}{c}\text { TYPE IV } \\
\text { HT }\end{array}$} & \multicolumn{2}{|c|}{ TYPE V } \\
\hline & $\mathbf{A}$ & B & $A^{d}$ & B & $A^{d}$ & B & & $A^{d}$ & B \\
\hline $\begin{array}{l}\text { Structural frame }{ }^{2} \\
\text { Including columns, girders, trusses }\end{array}$ & $3^{b}$ & $2^{b}$ & 1 & 0 & 1 & 0 & HT & 1 & 0 \\
\hline $\begin{array}{l}\text { Bearing walls } \\
\text { Exterior } f \\
\text { Interior }\end{array}$ & $\begin{array}{c}3 \\
3^{b}\end{array}$ & $\begin{array}{c}2 \\
2^{b}\end{array}$ & $\begin{array}{l}1 \\
1\end{array}$ & $\begin{array}{l}0 \\
0\end{array}$ & $\begin{array}{l}2 \\
1\end{array}$ & $\begin{array}{l}2 \\
0\end{array}$ & $\stackrel{2}{1 / \mathrm{HT}}$ & $\begin{array}{l}1 \\
1\end{array}$ & $\begin{array}{l}0 \\
0\end{array}$ \\
\hline $\begin{array}{l}\text { Nonbearing walls and partitions } \\
\text { Exterior } \\
\text { Interiore }\end{array}$ & & & & $\begin{array}{l}\text { See } \\
\text { See }\end{array}$ & $\begin{array}{l}602 \\
\text { n } 602\end{array}$ & & & & \\
\hline $\begin{array}{l}\text { Floor construction } \\
\text { Including supporting beams } \\
\text { and joists }\end{array}$ & 2 & 2 & I & 0 & 1 & 0 & HT & 1 & 0 \\
\hline $\begin{array}{l}\text { Roof construction } \\
\text { Including supporting beams } \\
\text { and joists }\end{array}$ & $11^{1} x^{c}$ & 1c & $1^{c}$ & $0^{c}$ & $j^{\circ}$ & 0 & HT & Ie & 0 \\
\hline
\end{tabular}




\section{Required Separation of Occupancies}

- For Mixed Occupancies fire separations are in accordance with Table 302.3.3 of IBC 2000

- For areas that are incidental to the main occupancy fire separations are in accordance with Table 302.1.1 of IBC 2000 


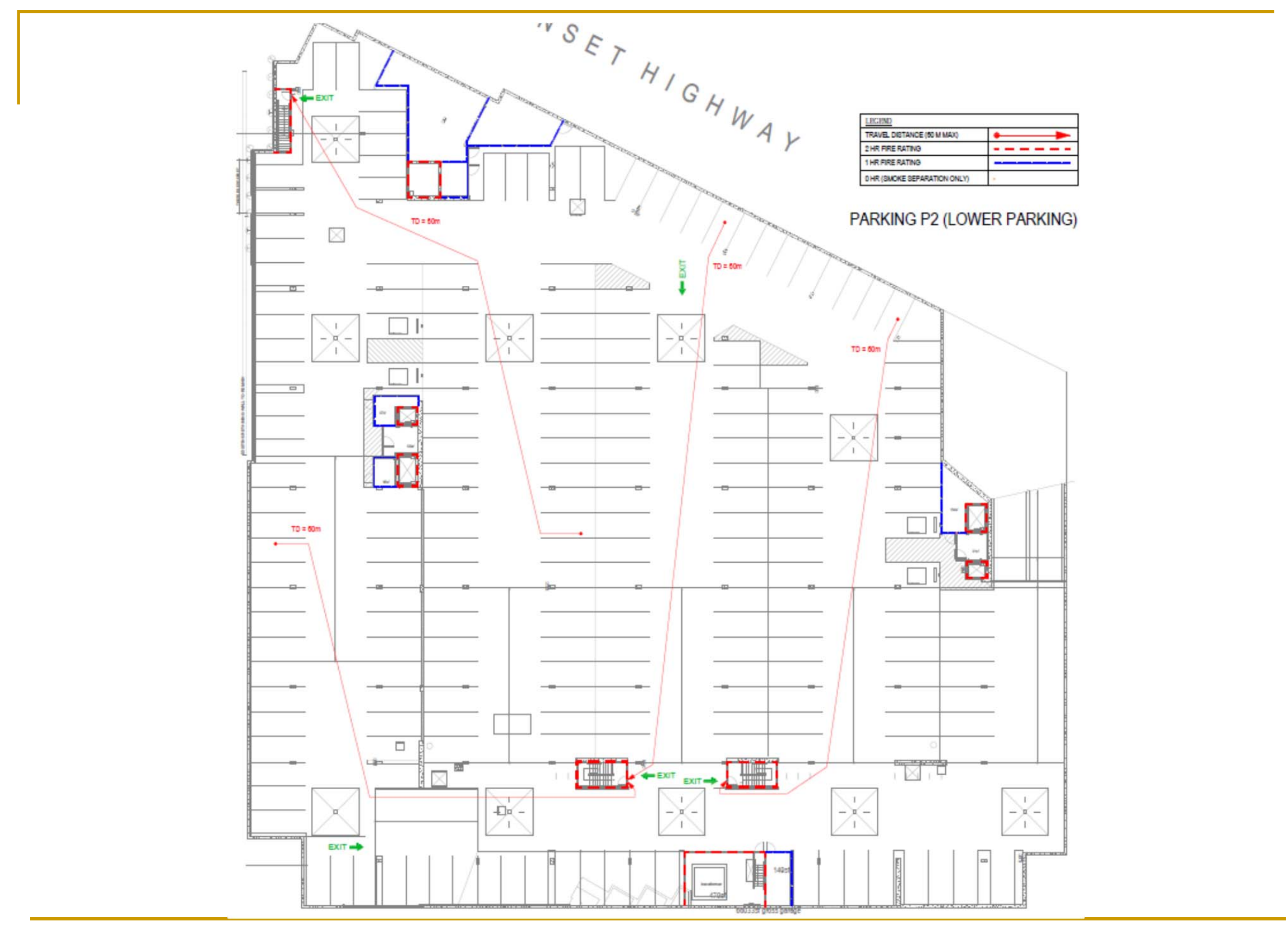




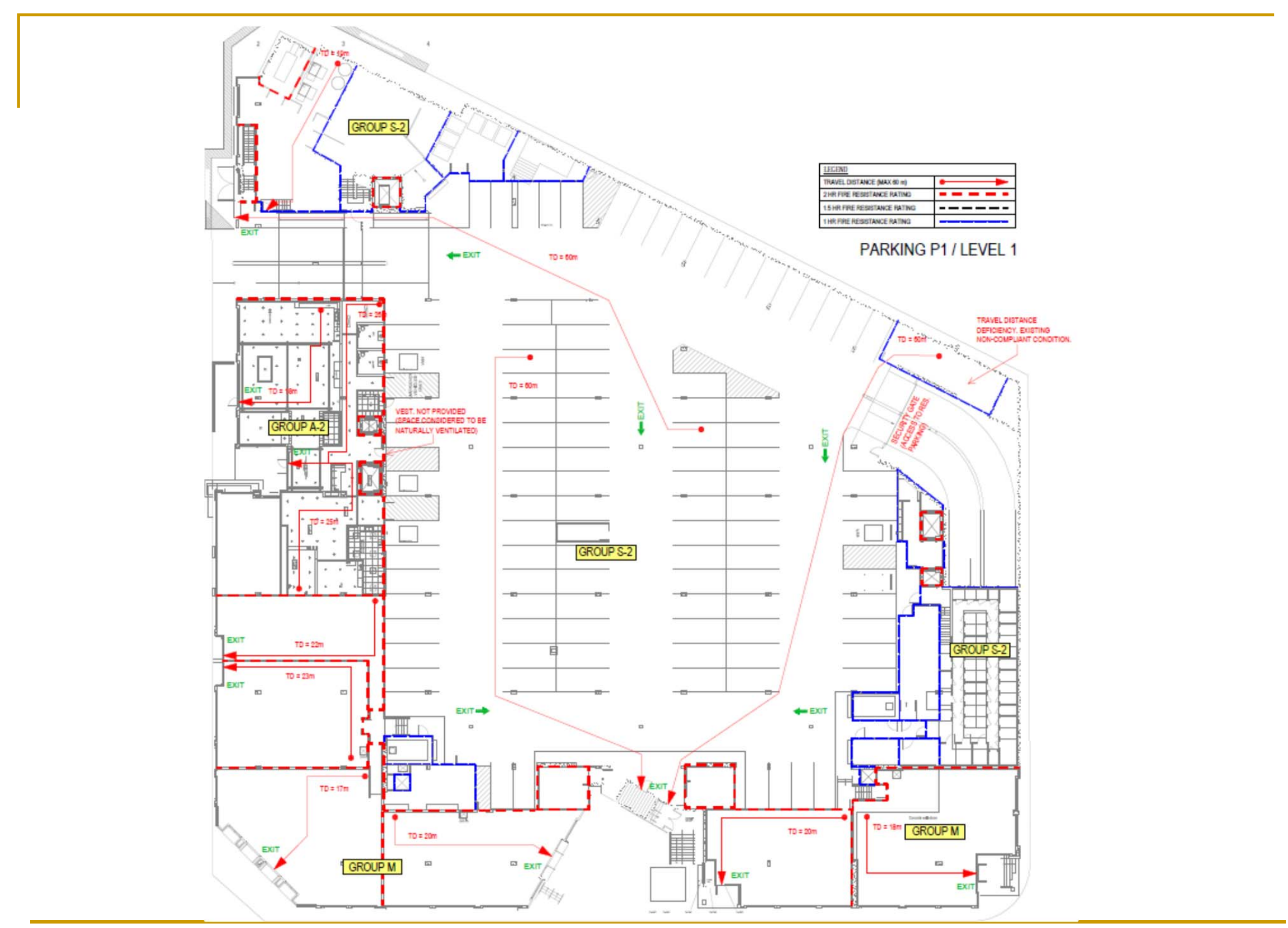




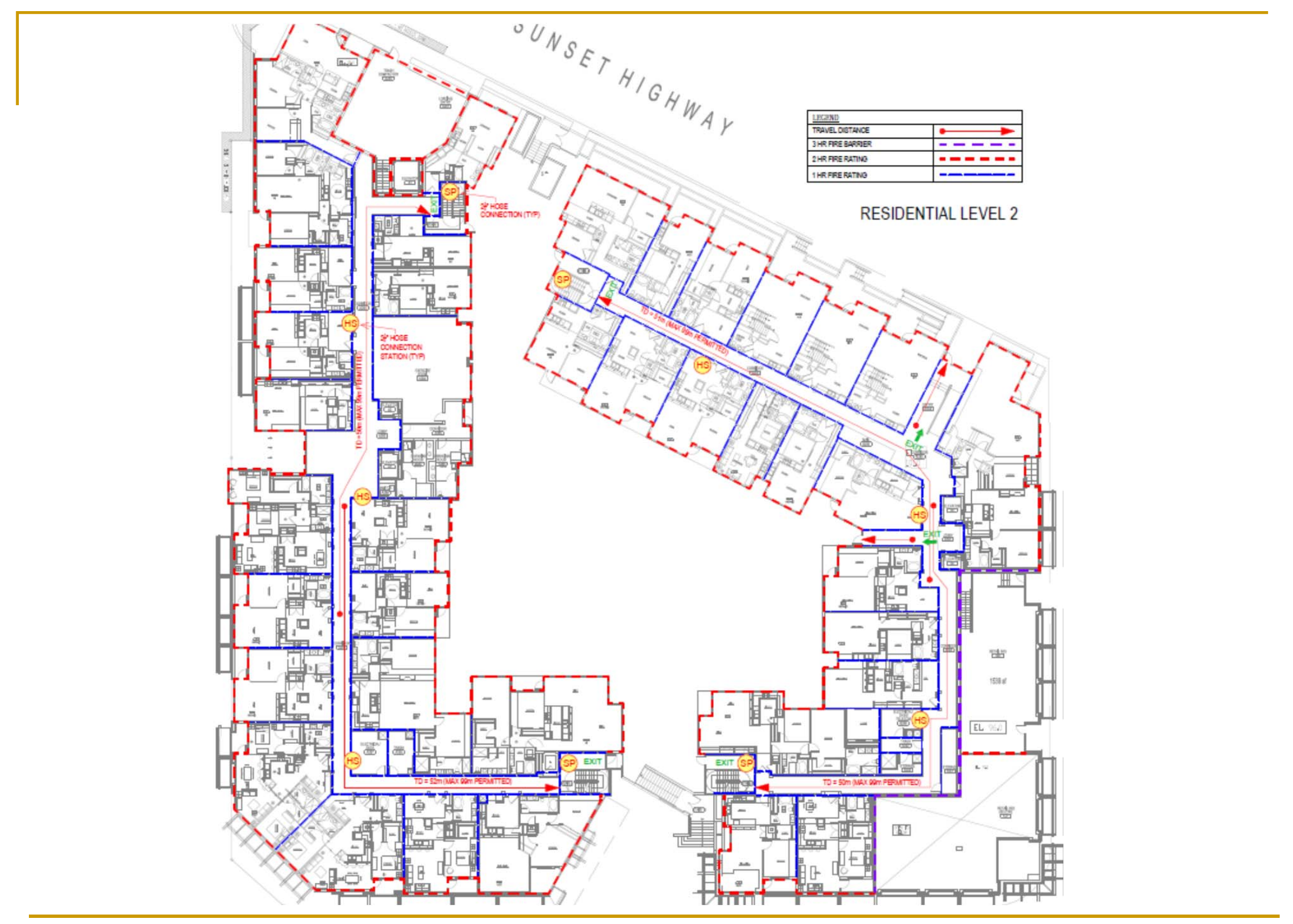




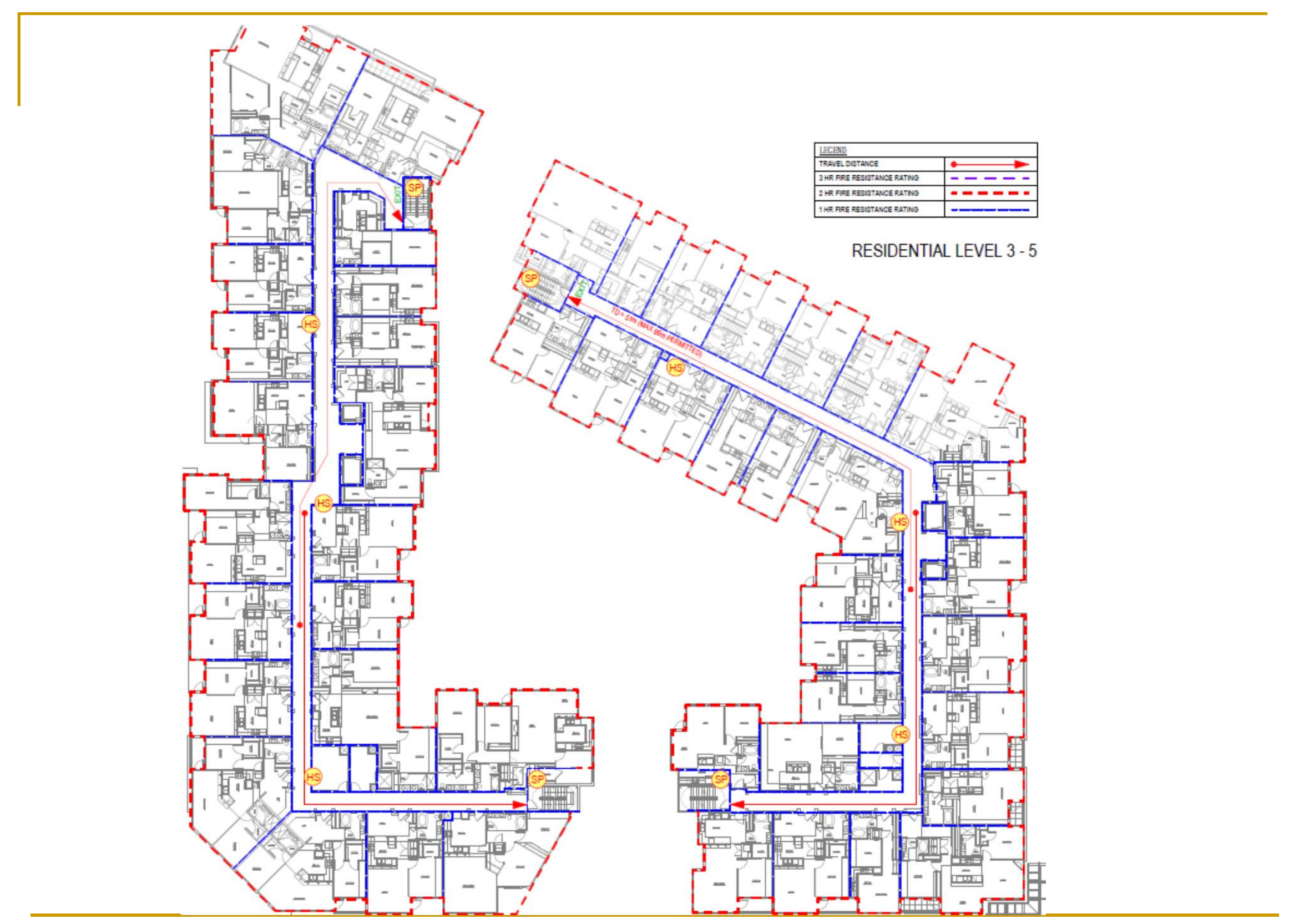




\section{Buildings Section}

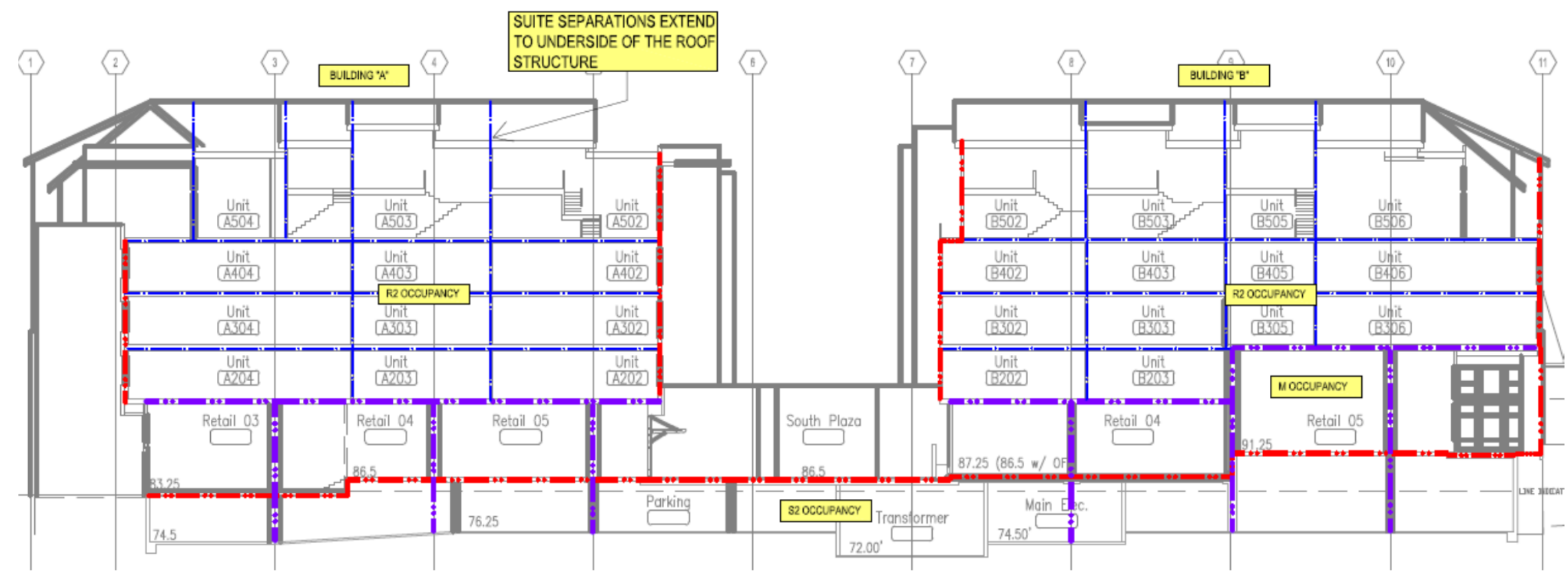




\section{Egress Analysis (Prescriptive) \\ Parking/Retail Building}

Table 1 - Parking Garage / Mercantile / Assembly (Building 1) LSC Table 7.3.3.1 Capacity Factors

\begin{tabular}{|c|c|c|c|c|}
\hline $\begin{array}{l}\text { Floor } \\
\text { Area }\end{array}$ & $\begin{array}{c}\text { Area } \\
\text { (sq. m) }\end{array}$ & $\begin{array}{c}\text { Occupant } \\
\text { Load Factor }\end{array}$ & $\begin{array}{l}\text { Capacity } \\
\text { (persons) }\end{array}$ & $\begin{array}{l}\text { Exit Capacity - persons } \\
\text { (metric) }\end{array}$ \\
\hline Perking P0 & 6130 & 18.6 gross & 330 & $\frac{434 \text { persons }}{(3 \times 1,100 \mathrm{~mm} \text { stairs @ } 7.6 \mathrm{~mm} / \text { person })}$ \\
\hline Parking P1 & 3677 & 18.6 gross & 198 & $\frac{365 \text { persons }}{(2 \times 914 \mathrm{~mm} \text { door } @ 5 \mathrm{~mm} / \text { person })}$ \\
\hline $\begin{array}{l}\text { Amenity } \\
\text { Area }\end{array}$ & 355 & 1.4 net & 253 & $\frac{365 \text { persons }}{(2 \times 914 \mathrm{~mm} \text { door } @ 5 \mathrm{~mm} / \text { person })}$ \\
\hline $\begin{array}{l}\text { Retail } \\
\text { - CRU1 } \\
\text { - CRU2 } \\
\text { - CRU3 } \\
\text { - CRU4 } \\
\text { - CRU5 } \\
\text { - CRU6 }\end{array}$ & $\begin{array}{l}125 \\
178 \\
212 \\
180 \\
150 \\
214\end{array}$ & $\begin{array}{l}2.8 \\
- \\
-- \\
-- \\
- \\
--\end{array}$ & $\begin{array}{l}45 \\
64 \\
76 \\
65 \\
54 \\
78\end{array}$ & $\begin{array}{l}\text { 365 persons per each CRU (typ) } \\
(2 \times 914 \mathrm{~mm} / \text { door } @ 5 \mathrm{~mm} / \text { person) }\end{array}$ \\
\hline
\end{tabular}




\section{Egress Analysis (Prescriptive)}

\section{Residential Building A (Similar to Bldg B)}

Table 2 - Building 2 (Residential Tower A) LSC Table 7.3.3.1 Capacity Factors

\begin{tabular}{|c|c|c|c|c|}
\hline $\begin{array}{l}\text { Floor } \\
\text { Area }\end{array}$ & $\begin{array}{l}\text { Area } \\
\text { (sq. m) }\end{array}$ & $\begin{array}{c}\text { Occupant } \\
\text { Load } \\
\text { Factor } \\
\text { (sq.m/per) }\end{array}$ & $\begin{array}{l}\text { Capacity } \\
\text { (persons) }\end{array}$ & $\begin{array}{l}\text { Exit Capacity } \\
\text { (metric) }\end{array}$ \\
\hline Level 2 & $\begin{array}{c}2297 \\
(21 \text { units) } \\
\end{array}$ & 18.6 & 124 & $\frac{365 \text { persons }}{(2 \times 914 \mathrm{~mm} \text { door } @ 5 \mathrm{~mm} / \text { person })}$ \\
\hline Level 3 & $\begin{array}{c}2297 \\
\text { (23 units) }\end{array}$ & 18.6 & 124 & $\frac{289 \text { persons }}{(2 \times 1,100 \mathrm{~mm} \text { stairs @ } 7.6 \mathrm{~mm} / \text { person })}$ \\
\hline Level 4 & $\begin{array}{c}2297 \\
\text { (23 units) }\end{array}$ & 18.6 & 124 & $\frac{289 \text { persons }}{(2 \times 1,100 \mathrm{~mm} \text { stairs @ } 7.6 \mathrm{~mm} / \text { person })}$ \\
\hline Level 5 & $\begin{array}{c}2297 \\
(23 \text { units) }\end{array}$ & 18.6 & 124 & $\frac{289 \text { persons }}{(2 \times 1,100 \mathrm{~mm} \text { stairs @ } 7.6 \mathrm{~mm} / \text { person })}$ \\
\hline Total & & & 496 & \\
\hline
\end{tabular}




\section{Phased Evacuation Concept (Smoke Control Measures)}

- Section 909 of IBC - set of requirements for limiting smoke movement between connected buildings

- Existing evacuation concept is on Figure 1

- Bldg A + Parking/Retail evacuate simultaneously

- No smoke protected vestibule for Bldg A

- Vestibule/Stair to Bldg A would require min. 12Pa pressure difference

- Fire Department will responds to Bldg A lobby

Figure 1: Existing

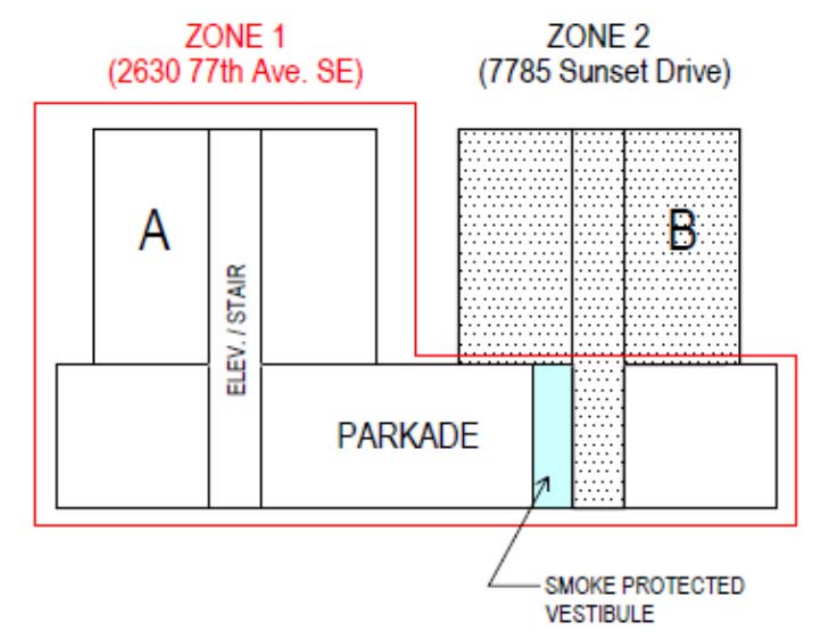

Figure 2: Alternative Arrangement

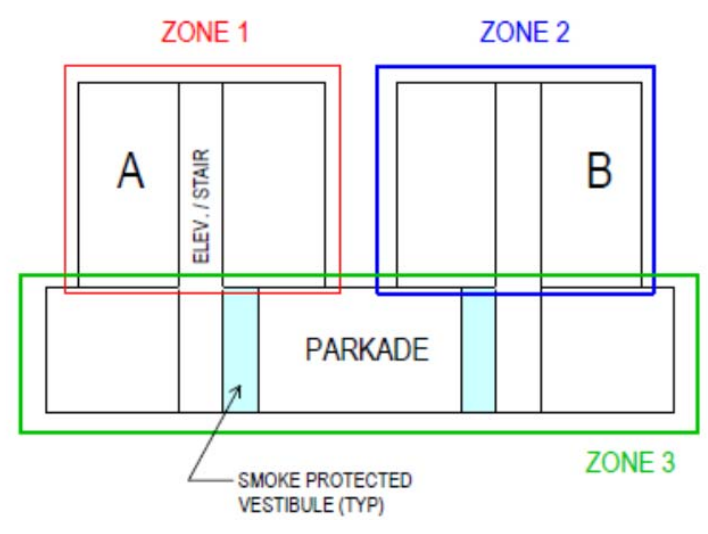




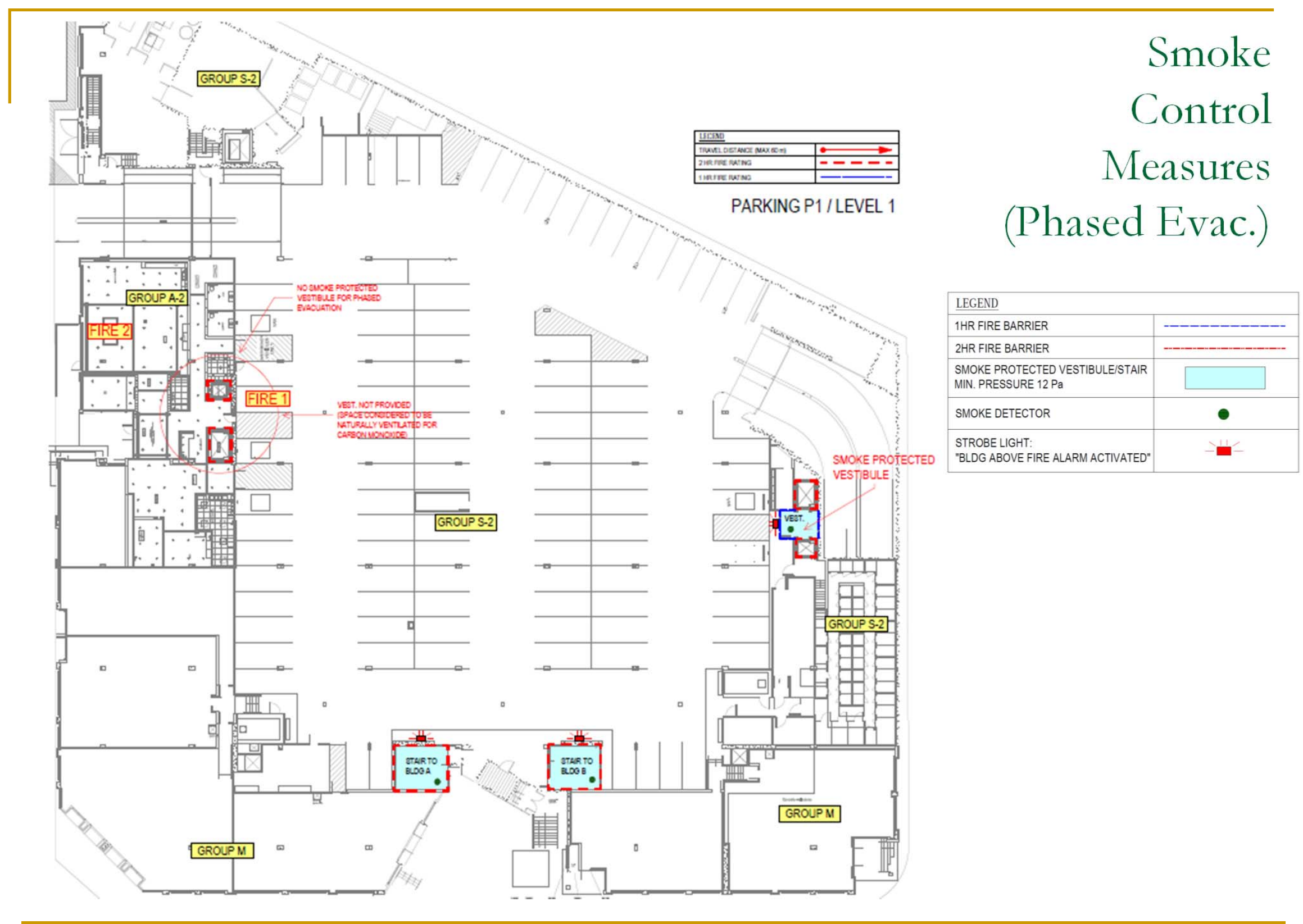




\section{Fire Protection Systems:}

\section{- Fire Sprinkler System}

- Water Supply: 85 psi static with 5000 gpm flow @ 20 psi residual pressure

- Parking/Retail Building

口 $\quad$ Parking $=\mathrm{OH} \mathrm{I}(0.15 \mathrm{gpm} / 1950 \mathrm{sq} . \mathrm{ft}) 30 \%$ remote area increase for dry system

- Storage $=\mathrm{OH} \mathrm{II} \mathrm{(0.20} \mathrm{gpm/1950} \mathrm{sq.ft)} \mathrm{30 \%} \mathrm{remote} \mathrm{area} \mathrm{increase} \mathrm{for} \mathrm{dry} \mathrm{system}$

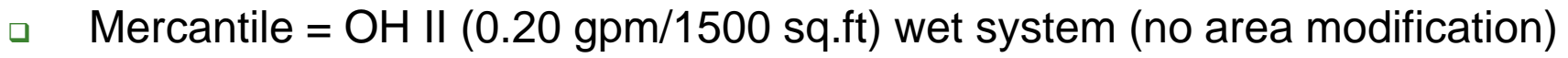

- Steel pipe - Dynaflow for mains \& dyna-thread for branchlines

- 6" Feed main for Bldg A \& B installed above insulation. Heat traced in one location between Bldg $A$ and $B$.

- Buildings $A$ and $B$

- Residential $=4$ sprinklers calculated at 0.10 density on Level 5

- CPVC plastic pipe (1" - 2" pipe)

- Pre-manufactured trash chute sprinklers (connect to system)

- Concealed floor joist spaces are not sprinklered (based on floor assembly)

- All outside balconies are sprinklered

- Building A \& B Attic Sprinkler System

- Tyco Attic Back-to-Back Sprinklers

- Seven sprinkler calculation per Manufacturer specification

- Dry system. Dynaflow for main \& dyna-thread for branchlines 


\section{Fire Sprinkler Hydraulic Calculations}

7700 Central

Remote Area: GARAGE \# 1

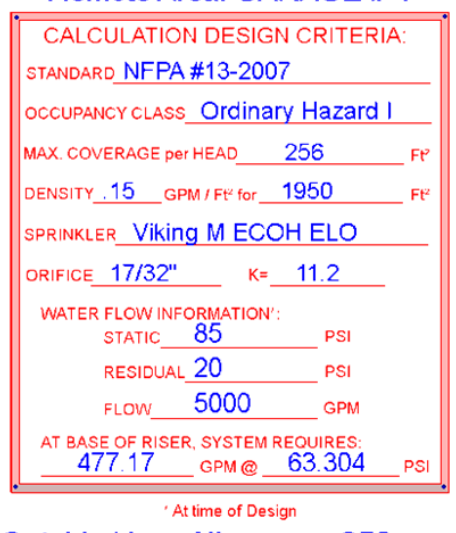

Outside Hose Allowance: $250 \mathrm{gpm}$ Safety Factor: +19.860 psi $(23.9 \%)$
7700 Central

Remote Area: RETAIL

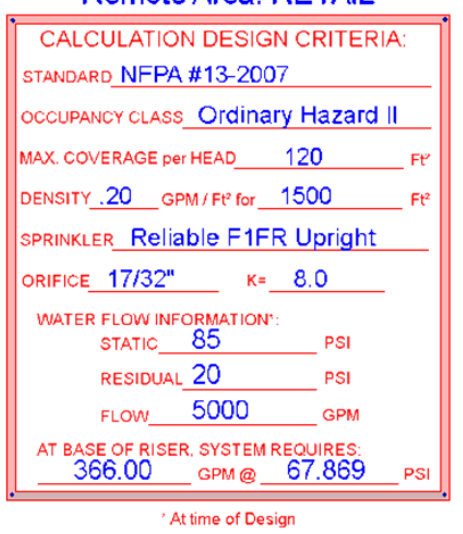

Outside Hose Allowance: $250 \mathrm{gpm}$ Safety Factor: +15.780 psi $(18.9 \%)$
7700 Central

Remote Area: STORAGE

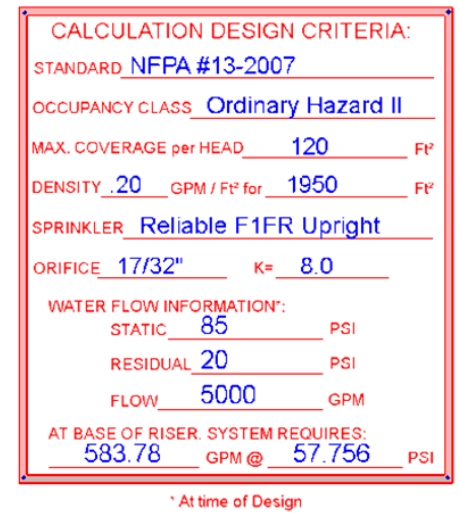

Outside Hose Allowance: $250 \mathrm{gpm}$ Safety Factor: +24.880 psi $(30.1 \%)$

7700 Central

Remote Area: Residential Unit

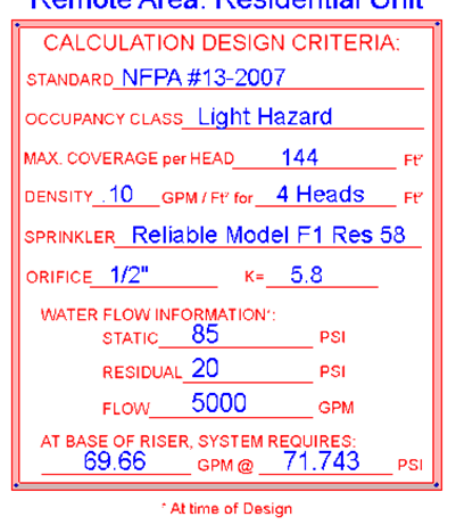

Outside Hose Allowance Req'd: $100 \mathrm{gpm}$ (LH) Outside Hose Allowance Added: $250 \mathrm{gpm}$ Safety Factor: +12.855 psi (15.2\%)

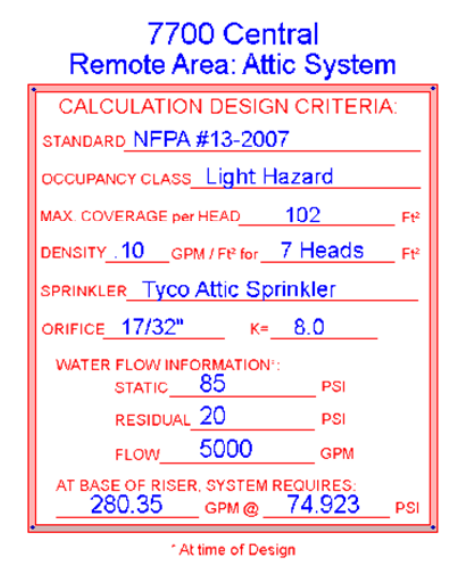

Outside Hose Allowance Req'd: 100 gpm (LH)

Outside Hose Allowance Added: $250 \mathrm{gpm}$ Safety Factor: +9.053 psi (10.8\%) 


\section{Fire Sprinkler Hydraulic Calculations}

\section{Central}

Remote Area: GARAGE \# 1

CALCULATION DESIGN CRITERIA:

STANDARD NFPA \#13-2007

OCCUPANCY CLASS Ordinary Hazard I

MAX. COVERAGE per HEAD $\quad 256$

DENSITY.15 GPM/Ft' for 1950 $\mathrm{Fr}^{\mathrm{P}}$

SPRINKLER Viking M ECOH ELO

ORIFICE 17/32"

$\mathrm{K}=11.2$

WATER FLOW INFORMATION':

$\begin{array}{lll}\text { STATIC } \frac{85}{8} & \text { PSI } \\ \text { RESIDUAL } 20 & \\ \text { PSI } & \\ \text { FLOW } 5000 & \text { GPM } & \\ \text { SEOF RISER, SYSTEM REQUIRES: } & \\ 77.17 & \text { GPM @ } 63.304 & \text { PSI }\end{array}$

'At time of Design

Outside Hose Allowance: $250 \mathrm{gpm}$

Safety Factor: +19.860 psi $(23.9 \%)$
7700 Central

\section{Remote Area: STORAGE}

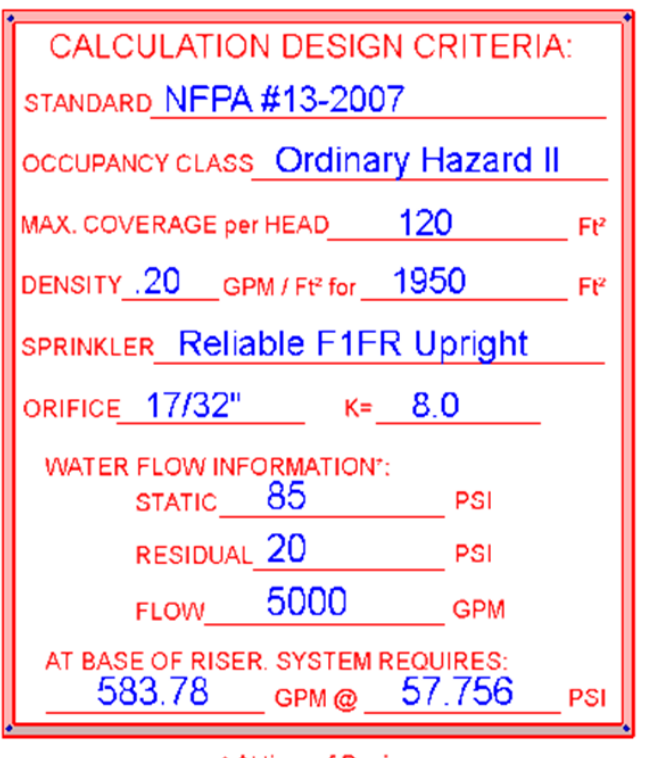

At time of Design

Outside Hose Allowance: $250 \mathrm{gpm}$ Safety Factor: +24.880 psi (30.1\%) 


\section{Fire Protection Systems:}

\section{- Parking P1/Level 1 Distribution piping}

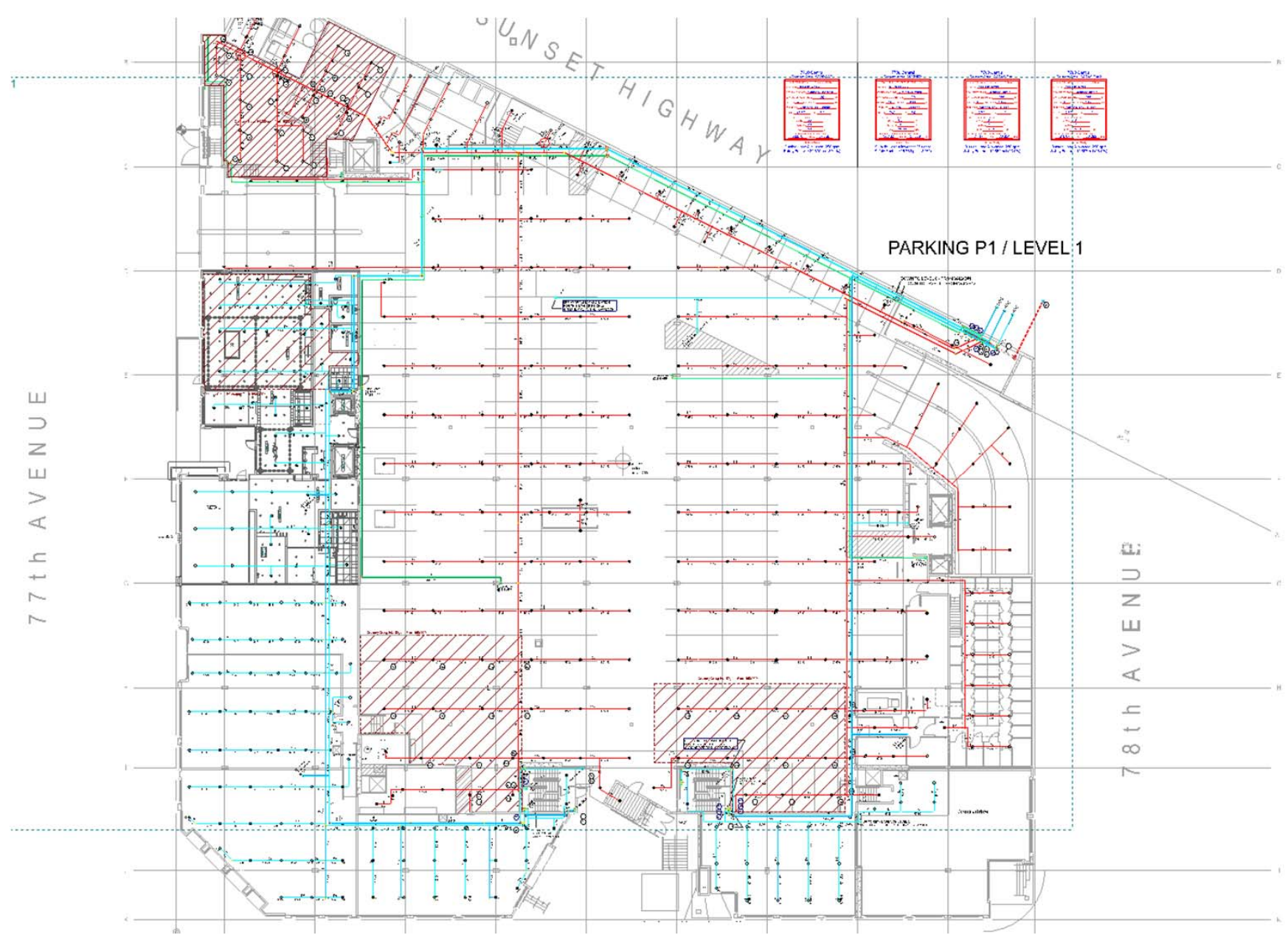




\section{Residential Level 2 Sprinkler System}

SE Enlarged view

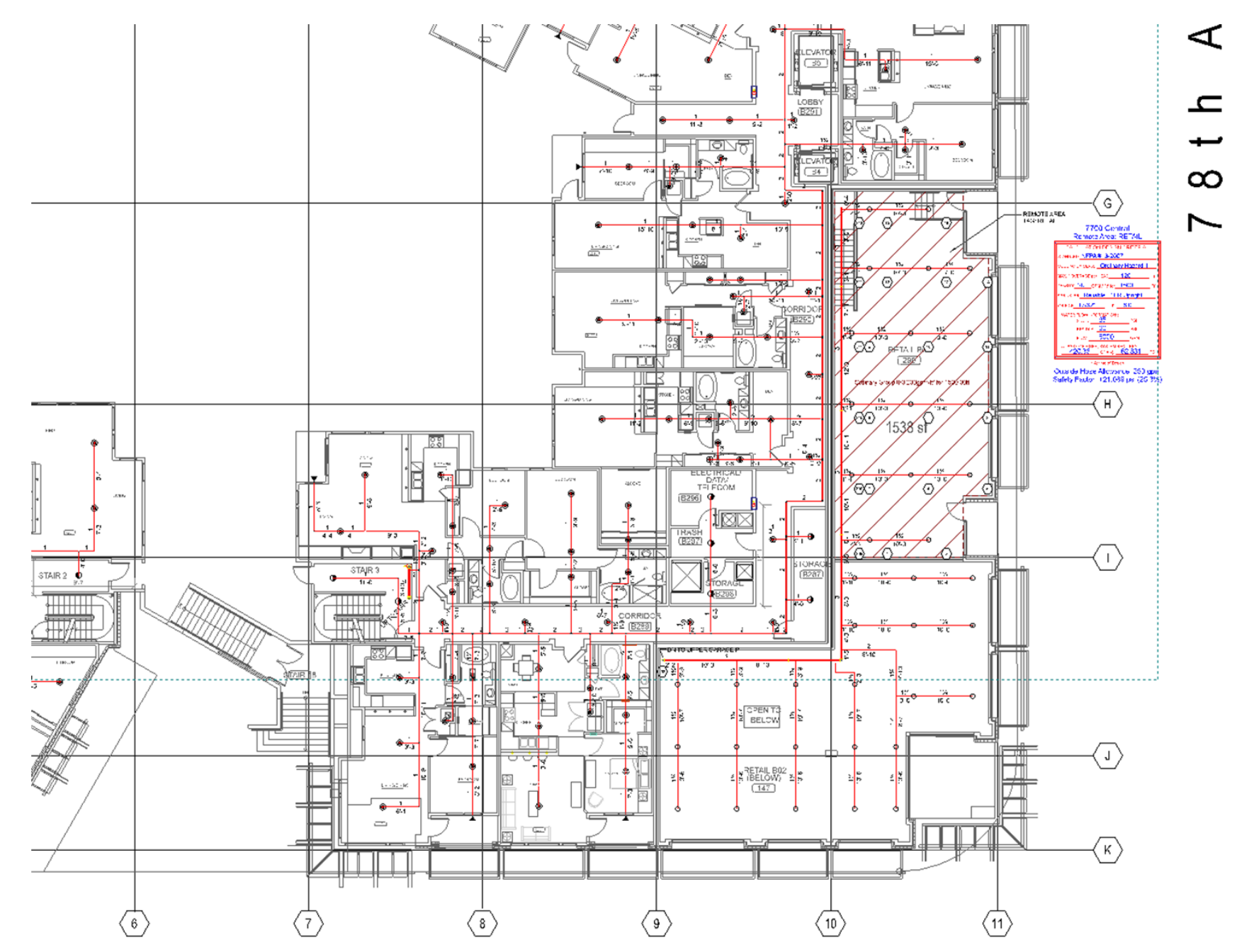




\section{Attic Sprinklers. Tyco Back-to-Back}

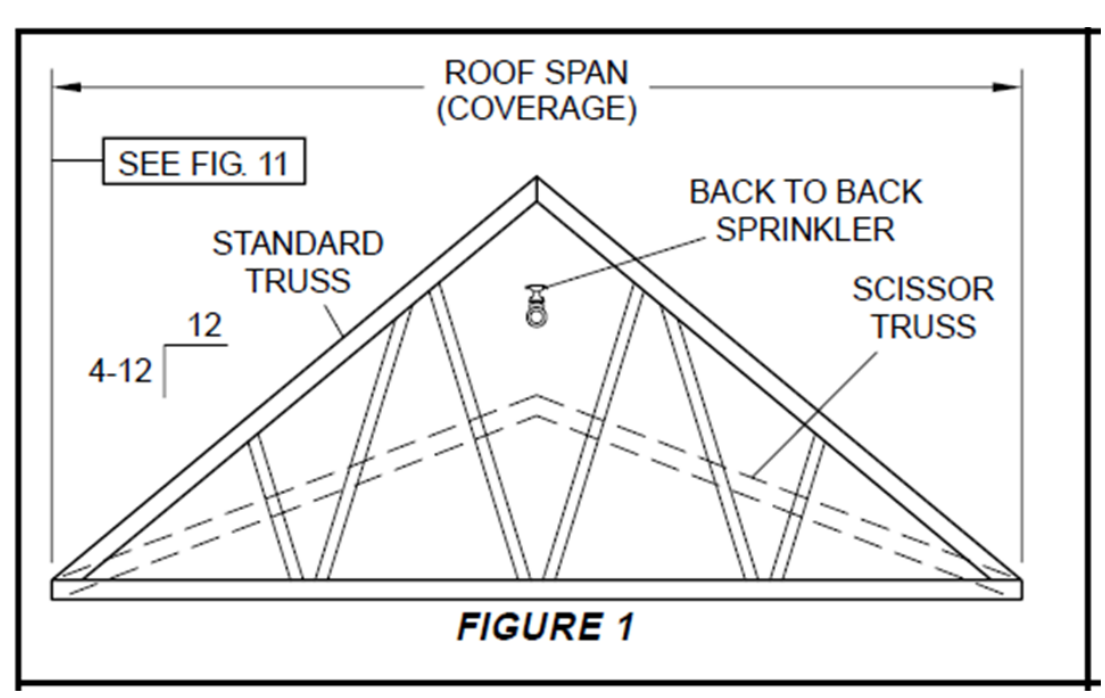

- Dual directional specialty sprinkler that throws a narrow, but long pattern in two directions.

- Very efficient attic system design (vs. standard area/density method)

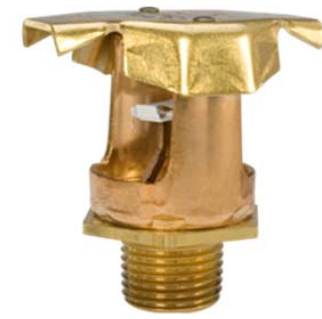




\section{Fire Protection Systems:}

\section{- Fire Alarm}

- The development is considered as a single building for fire alarm system installation

- A single-stage fire alarm system is provided

- Manual pull stations are installed at every floor near every exit and near the principal entrances to buildings.

- Smoke detectors are provided in each exit stairway, in public corridors in residential portions of the development and in all dwelling unit bedrooms and outside of bedrooms.

- Smoke detector in Smoke Protected Vestibule

- The fire alarm system will automatically transmit alarm signals and supervisory signals to the Fire Department.

- Bldg A + Parking/Retail $=263077^{\text {th }}$ Ave SE

a $\quad$ Bldg $B=7785$ Sunset Drive

- Emergency generator will supply power for approximately 90 minutes. (IBC 1003.2.11.2)

- Phased Evacuation (Smoke Protected Vestibules) - discussed earlier in Exit Systems

- Bldg A + Parking/Retail

ㅁ Bldg B 


\section{Performance-Based Approach Outline:}

- Scenario \# 1

- Garage Automobile Fire

- Scenario \# 2

- Amenity Room Fire

- Scenario \#3

- Level 5 Mezzanine Kitchen Fire

Approach: $\quad$ Scenario-based analysis (design fires) will be used.

Tenability: $\quad 10 \mathrm{~m}$ visibility and $60 \mathrm{deg}$. C temperature

FDS setup: $\quad$ All surfaces are modeled as "inert" (do not absorb any heat)

Design Fire :

1. Heat Release Rate (t-squared fire) UON

2. Smoke production rate (Propane is a default setting in FDS)

- For more accurate fire scenarios soot yields appropriate for each fire should be selected.

$\square \quad$ My analysis is based on the default value (propane) 


\section{Scenario \#1 (Garage Automobile Fire)}

- SFPE Handbook $4^{\text {th }}$ Edition. Pg 3-41. Figure 3-1.79 Data from FRS and VTT laboratories

- $\quad$ NFPA 101 (2003) Ch. 5 Section 5.5.3.8

Design Fire Scenario \# 8

- This scenario addresses concerns regarding unreliability of the fire protection systems. One system must be disabled.

- $\quad$ Fire resistance rating between the parking garage and the Amenity space will be compromised (open door)

- Sprinkler system will remain active.

- $\quad$ NFPA 101 (2003) Ch. 5 Section 5.2

Performance Criteria

a Method 4-must demonstrate that no fire

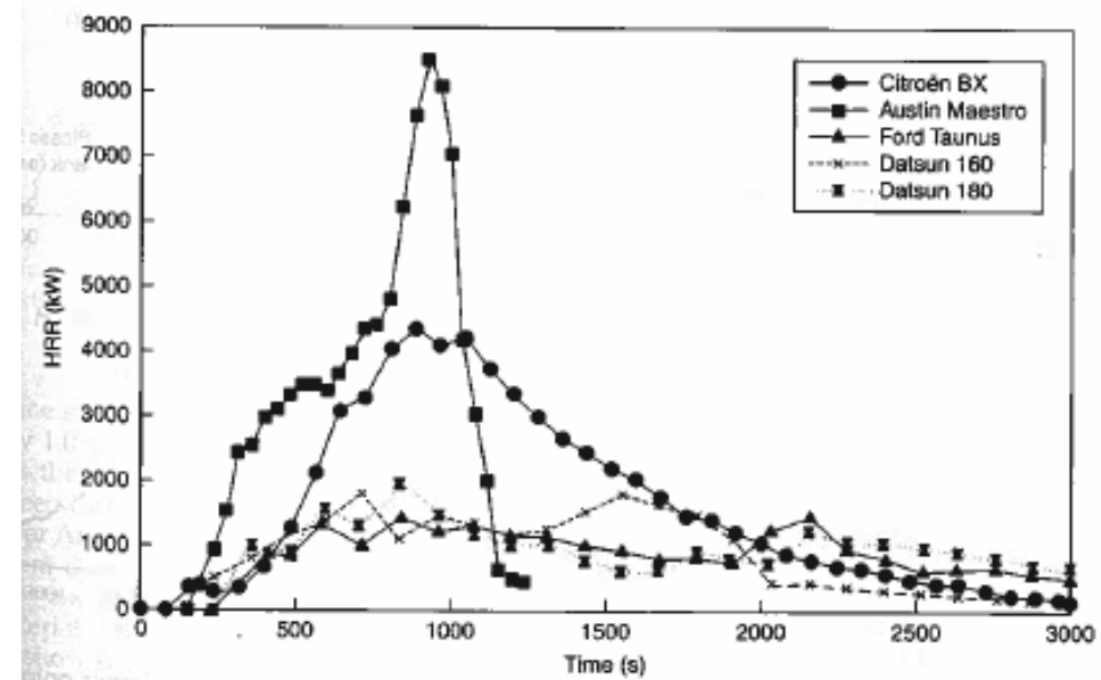
effects will reach any occupied room. Very conservative approach.

- Advantage - no need to account for occupant movement, location or pre-fire characteristics.

- $\quad \mathrm{HRR}=6 \mathrm{MW}$ (car fire)

口 Assumed steady state fire 


\section{Scenario \# 1 cont. (FDS results)}

- Steady state fire - early sprinkler activation does not have any effect on smoke development in FDS, on temp. only

- Parking garage area was confined for modeling purposes (very conservative)

- High ceiling height in the Parking garage $(4 \mathrm{~m})$ reduces smoke migration to the Amenity

- This scenario meets the selected Tenability Criteria and LSC Method 4. RSET analysis is not required.

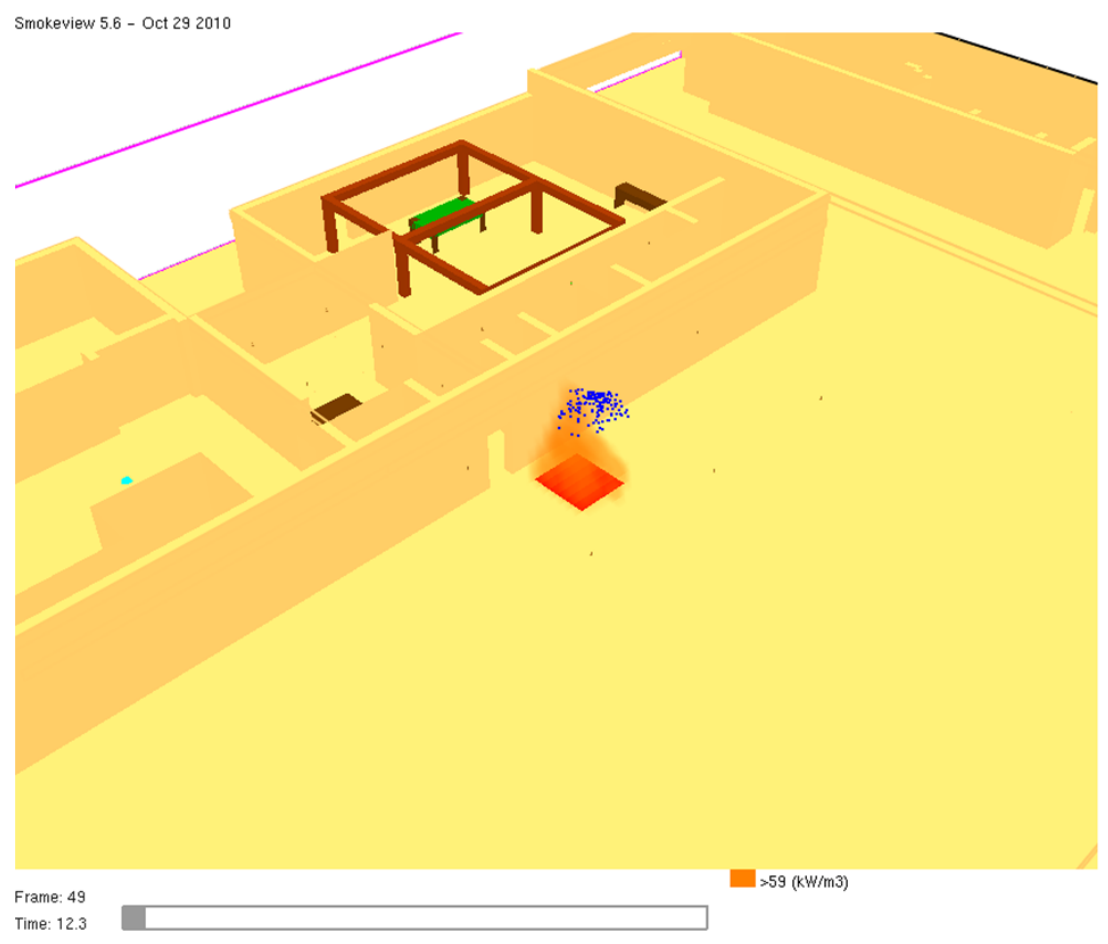
Smokeview 5.6 - Oct 292010

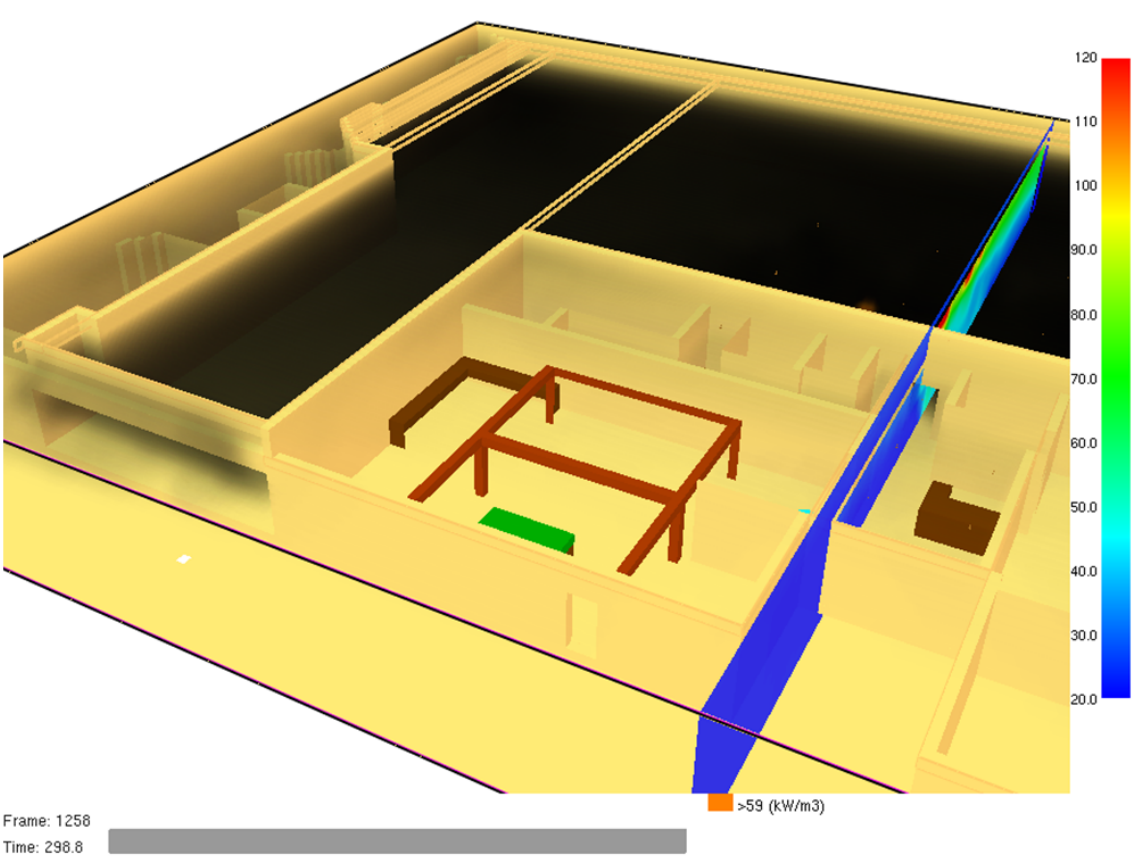




\section{Scenario \#2 (Amenity Room Fire)}

- SFPE Handbook $4^{\text {th }}$ Edition. Pg 3-48. Figure 3-1.102 Data from NIST.

- NFPA 101 (2003) Ch. 5 Section 5.5.3.8 Design Fire Scenario \# 8

- This scenario addresses concerns regarding unreliability of the fire protection systems. One system must be disabled.

- $\quad$ Sprinkler system will NOT be active.

- NFPA 101 (2003) Ch. 5 Section 5.2 Performance Criteria

- Method 2 - must meet tenability criteria for visibility and temperature until all occupant safely evacuate

- ASET vs. RSET will be required

- $\mathrm{HRR}=3 \mathrm{MW}$ (Upholstered furniture)

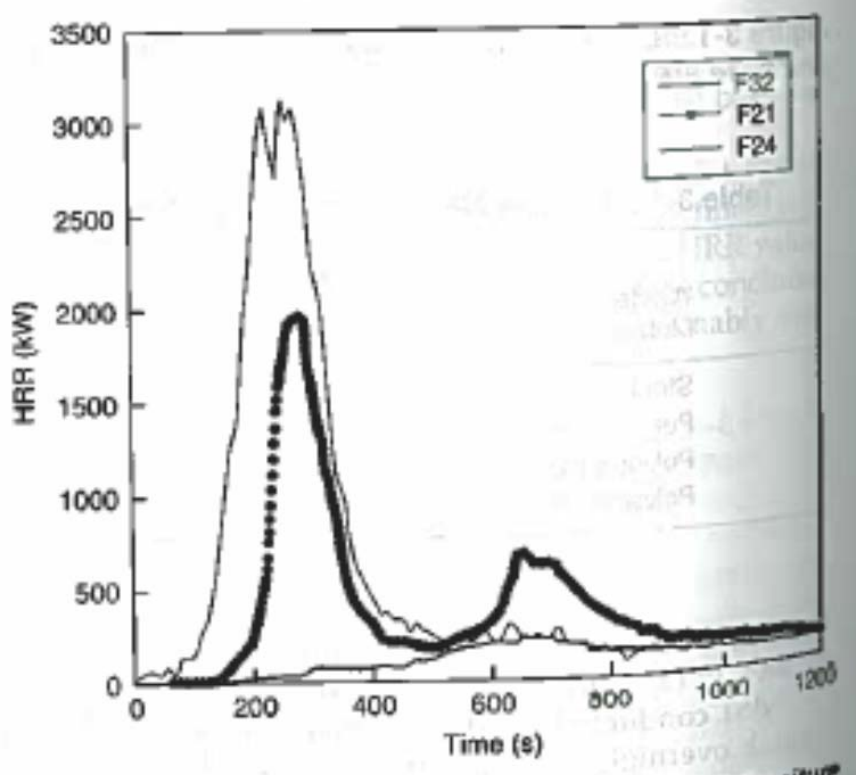

Figure 3-1.102. HRR of several upholstered fumilure items tested at NIST. ${ }^{2}$

- Fast growth rate fire

- Fire will compromise one of the exits out of the Amenity space 


\section{Scenario \# 2 cont. (FDS setup)}

- $\quad$ Fire located in the corner of the room

- Visually open space

- 1 exit will be compromised
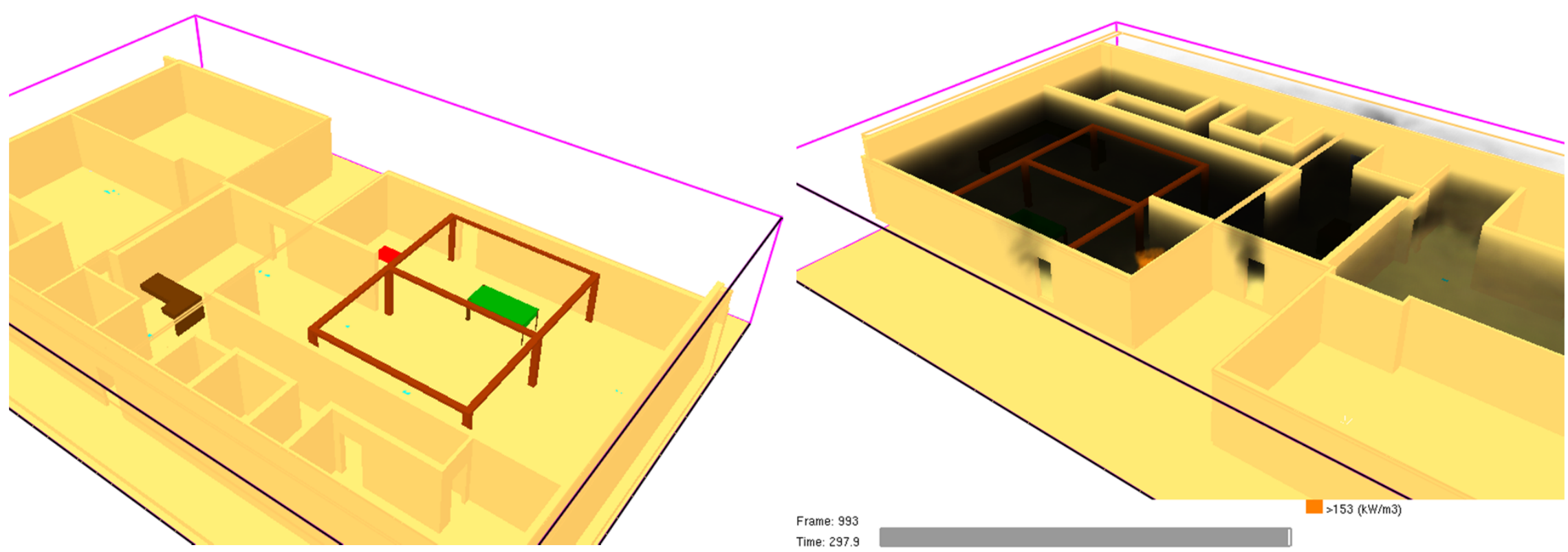


\section{Scenario \# 2 cont. (FDS results - ASET)}

- Visibility criteria of $10 \mathrm{~m}$ is reached at approximately $165 \mathrm{sec}$. (2.75 min)

- Maximum temperature criteria of $60 \mathrm{deg} . \mathrm{C}$ is reached at approximately $125 \mathrm{sec}$. (2.08 min)

- Most conservative time of $125 \mathrm{sec}$. (2.08 min) will be used for ASET.

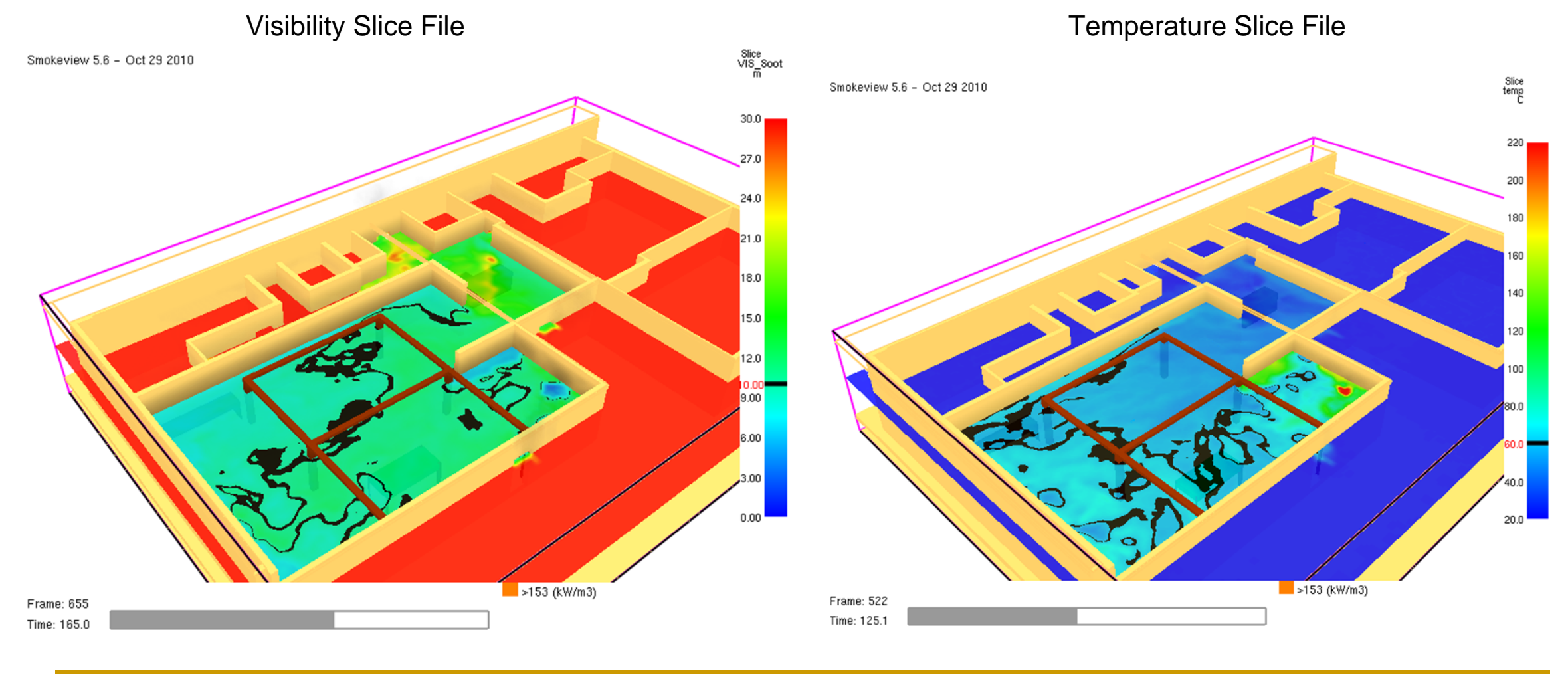




\section{RSET (Amenity Room Fire)}

- Fire alarm activation

- Visually open space

- $\quad 10 \mathrm{sec}$ (pull station activated)

- Pre-movement time

- Table 3-12.1 of SFPE Handbook (4 $4^{\text {th }}$ Edition) NFPA Timetable of Station Nightclub Fire

- $19 \mathrm{sec}$. - crowd begins to react.

- Movement Time:

- $\quad 91.5$ seconds based on Pathfinder (Steering mode)

- 259 occupant load

口 2 exits only (1 is compromised)

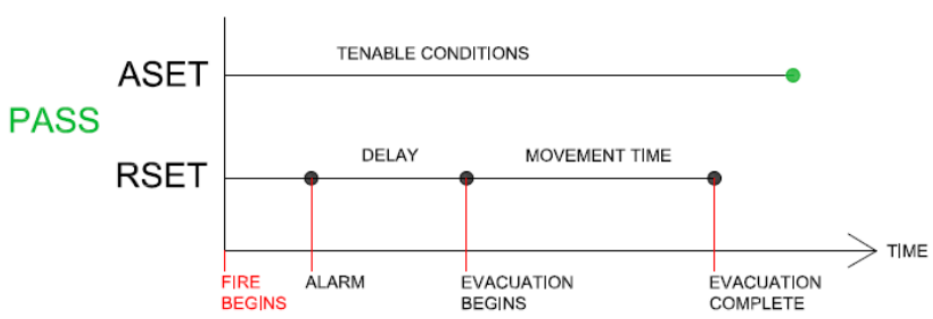

a Sprinklers are not active

- RSET Time $=120.5 \mathrm{sec}$.

- Safety factor built into the tenability criteria (compromised exit)

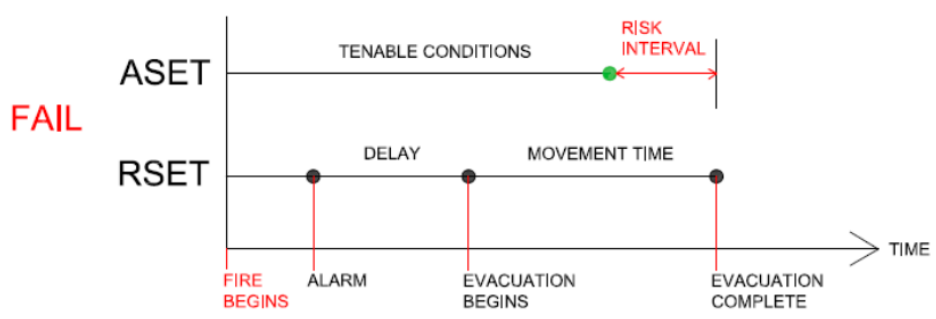




\section{Scenario \# 2 cont. (ASET vs RSET)}

- $\quad$ ASET $=125 \mathrm{sec}$. (2.08 $\mathrm{min})$ based on FDS simulation

- RSET $=120.5 \mathrm{sec}$. $(2.0 \mathrm{~min})-1$ exit door is compromised

- $\quad$ ASET > RSET (MoS - built into the Tenability Criteria. 1 exit compromised)

- Performance objective is met

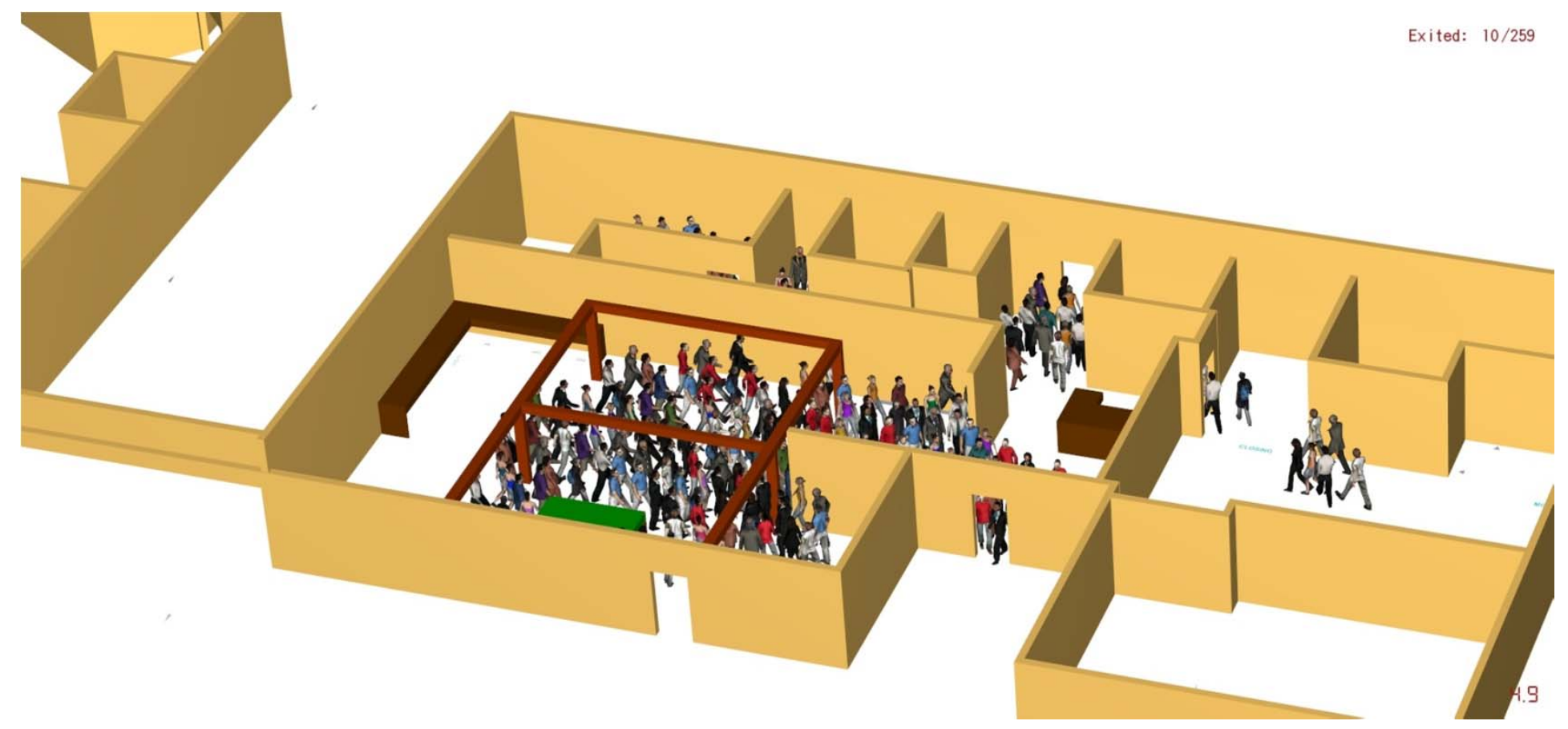




\section{Scenario \#3 (Level 5 Residential Kitchen Fire)}

- HRR data for Kitchen fire is from paper by NIST.

- NFPA 101 (2003) Ch. 5 Section 5.5.3.8 Design Fire Scenario \# 8

- Sprinkler system will NOT be active

- Smoke detector at mezzanine level

- NFPA 101 (2003) Ch. 5 Section 5.2

Performance Criteria

- Method 2 - must meet tenability criteria for visibility and temperature until all occupant safely evacuate

- $\quad$ ASET vs. RSET will be required

- $\quad$ HRR $=700 \mathrm{~kW}$ (Pan fire)

- Account for possibility of multiple pans catching on fire

- Fast growth rate fire

\section{UL 300A Fire Characterization}

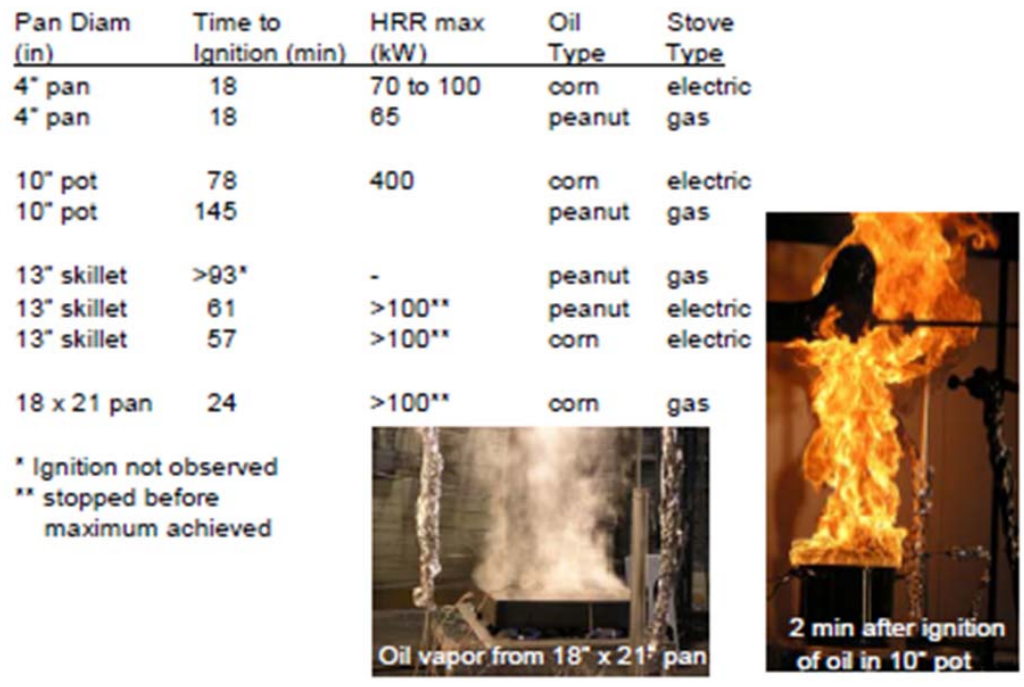




\section{Scenario \# 3 cont. (FDS results - ASET)}

- $\quad$ Kitchen fire $(700 \mathrm{~kW})$

- Smoke detector at mezzanine level activates at approximately 36 seconds

- Tenability temperature limit on main level is reached at approximately 145 seconds @ elevation of $1.8 m$

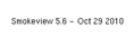

Smokeview 5.6 - oct 292010
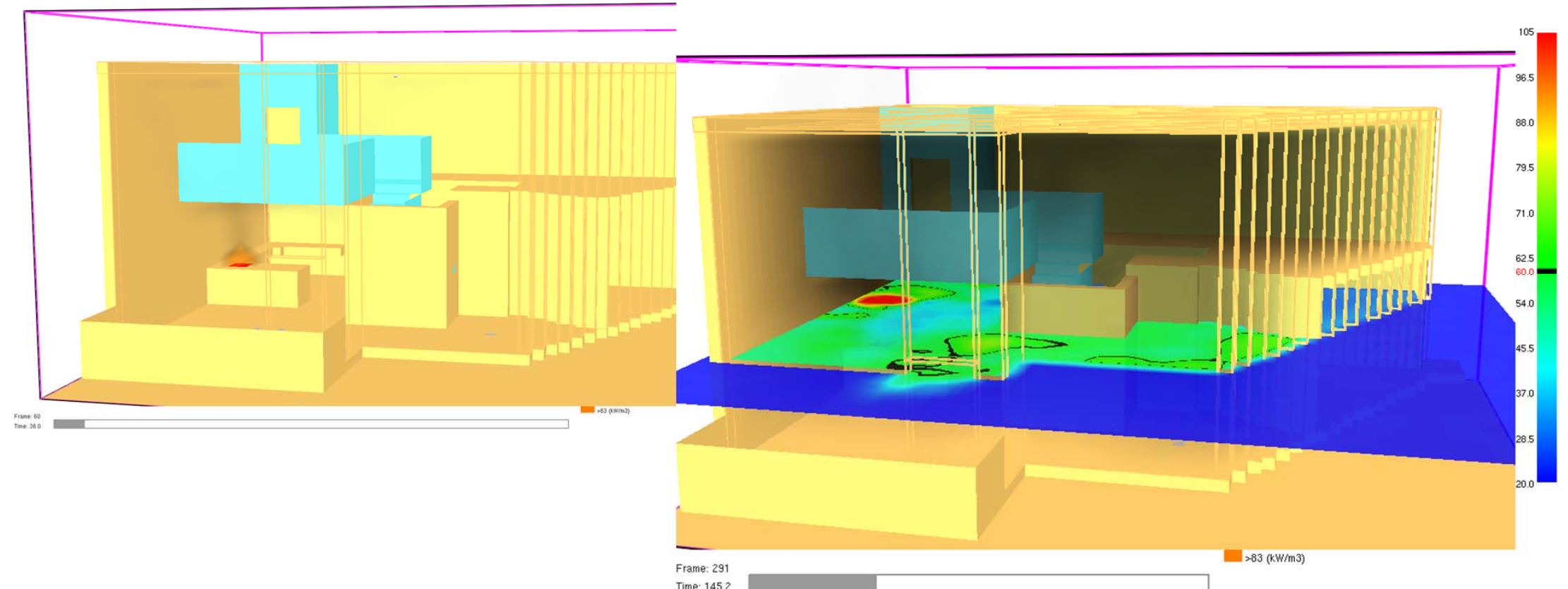


\section{RSET (Mezzanine Kitchen Fire)}

- Smoke detector activation

- $36 \mathrm{sec}$.

- Pre-movement time

- $60 \mathrm{sec}$. (reasonable estimation)

- Movement Time:

- $\quad 15.5$ seconds based on Pathfinder (Steering mode)

- 12 persons occupant load

- 1 exits

- Sprinklers are not active

- RSET Time $=111.5 \mathrm{sec}$.

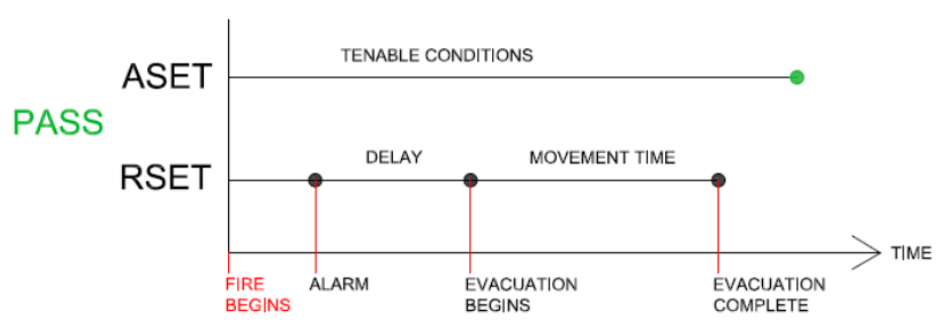

- Safety factor built into the tenability criteria (large HRR)

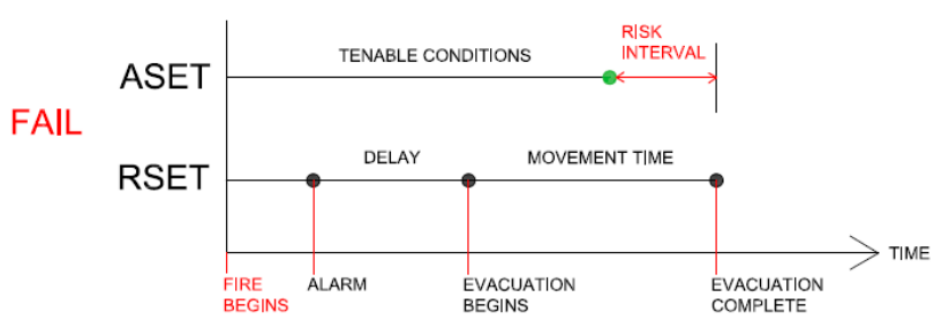




\section{Scenario \# 3 cont. (ASET vs RSET)}

- $\quad$ ASET $=145 \mathrm{sec} .(2.41 \mathrm{~min})$

- $\quad$ RSET $=111.5 \mathrm{sec} .(1.9 \mathrm{~min})$

- $\quad$ ASET > RSET x (MoS - built into Tenability Criteria. Large HRR)

- Performance objective is met

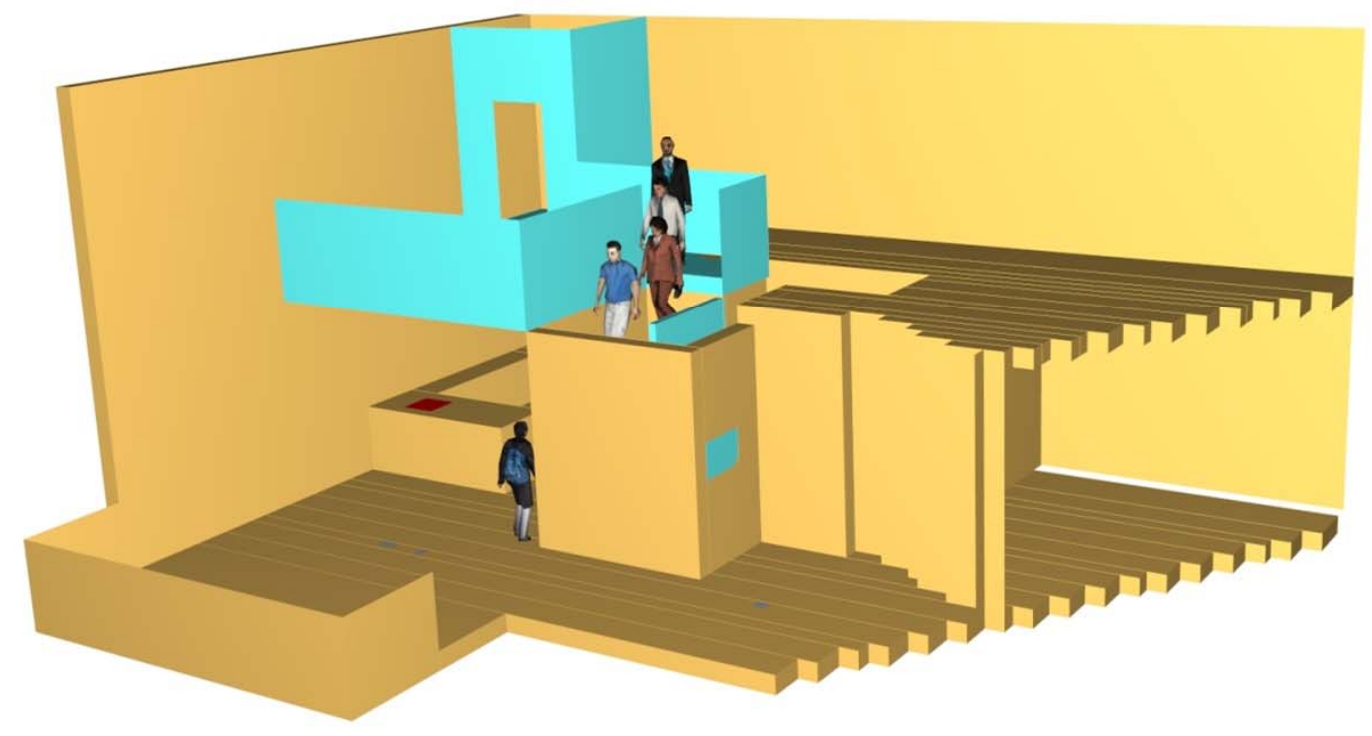




\section{Recommendations:}

$\underline{\text { Item } 1}$

- $45 \%$ of unprotected openings are permitted per Table 704.3 (Exceeded)

a Further review of the architectural elevation drawings are needed to verify

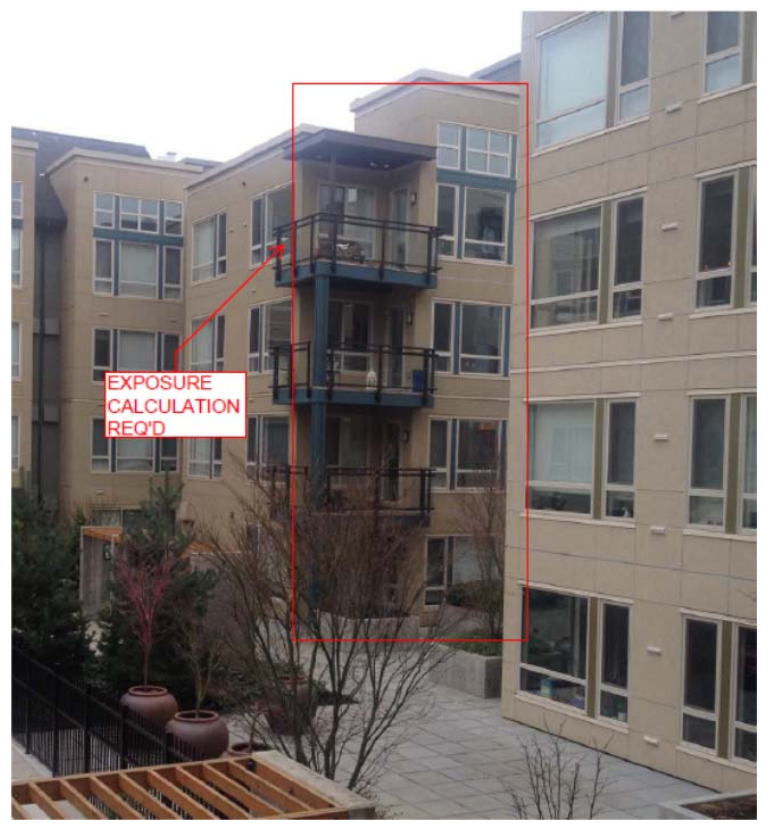




\section{Recommendations:}

\section{Item 2}

- Travel distance deficiency on the parking level P1.

- $60 \mathrm{~m}$ (200 ft) travel distance is exceeded from the Sprinkler Riser Room

\section{Item 3}

- Garbage room door (1 hr rated closure) in the garage was propped open during my site visit. This appears to be typical during the regular business hours. 


\section{Thank you!}

Comments or questions? 